\title{
Field-dependent dynamic responses from dilute magnetic nanoparticle dispersions
}

\author{
Fock, Jeppe; Balceris, Christoph ; Costo, Rocio; Zeng, Lunjie; Ludwig, Frank; Hansen, Mikkel Fougt
}

Published in:

Nanoscale

Link to article, DOI:

10.1039/C7NR07602A

Publication date:

2018

Document Version

Peer reviewed version

Link back to DTU Orbit

Citation (APA):

Fock, J., Balceris, C., Costo, R., Zeng, L., Ludwig, F., \& Hansen, M. F. (2018). Field-dependent dynamic responses from dilute magnetic nanoparticle dispersions. Nanoscale, 10, 2052-2066.

https://doi.org/10.1039/C7NR07602A

\section{General rights}

Copyright and moral rights for the publications made accessible in the public portal are retained by the authors and/or other copyright owners and it is a condition of accessing publications that users recognise and abide by the legal requirements associated with these rights.

- Users may download and print one copy of any publication from the public portal for the purpose of private study or research.

- You may not further distribute the material or use it for any profit-making activity or commercial gain

- You may freely distribute the URL identifying the publication in the public portal 
This article can be cited before page numbers have been issued, to do this please use: J. Fock, C. Balceris, R. Costo, L. Zeng, F. Ludwig and M. F. Hansen, Nanoscale, 2017, DOI: 10.1039/C7NR07602A.

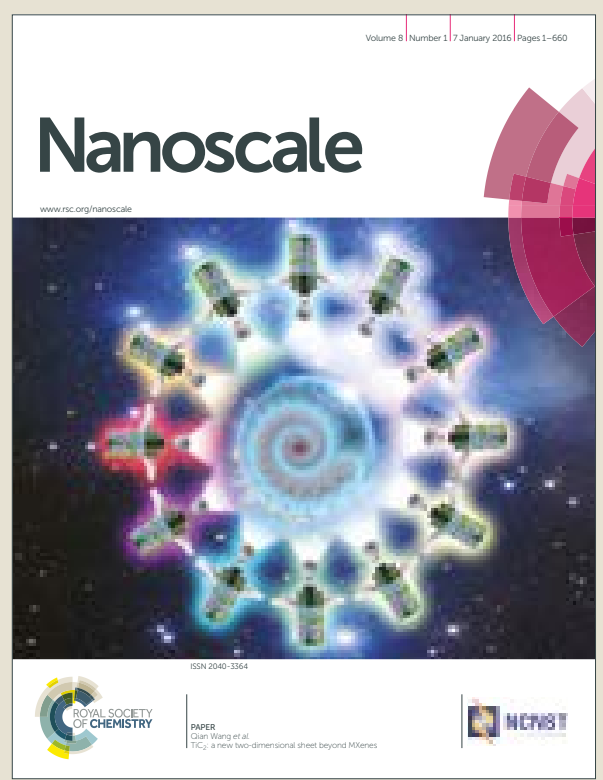

This is an Accepted Manuscript, which has been through the Royal Society of Chemistry peer review process and has been accepted for publication.

Accepted Manuscripts are published online shortly after acceptance, before technical editing, formatting and proof reading. Using this free service, authors can make their results available to the community, in citable form, before we publish the edited article. We will replace this Accepted Manuscript with the edited and formatted Advance Article as soon as it is available.

You can find more information about Accepted Manuscripts in the author guidelines.

Please note that technical editing may introduce minor changes to the text and/or graphics, which may alter content. The journal's standard Terms \& Conditions and the ethical guidelines, outlined in our author and reviewer resource centre, still apply. In no event shall the Royal Society of Chemistry be held responsible for any errors or omissions in this Accepted Manuscript or any consequences arising from the use of any information it contains. 


\section{ARTICLE TYPE}

Cite this: DOI: $10.1039 /$ xxxxxxxxxx

Accepted Date

DOI: 10.1039/xxxxxxxxxx www.rsc.org/journalname

\section{Field-dependent dynamic responses from dilute mag- netic nanoparticle dispersions ${ }^{\dagger}$}

\author{
Jeppe Fock, ${ }^{a}$ Christoph Balceris, ${ }^{b}$ Rocio Costo, ${ }^{c}$ Lunjie Zeng, ${ }^{d}$ Frank Ludwig, ${ }^{b}$ and Mik- \\ kel Fougt Hansen $* a$
}

The response of magnetic nanoparticles (MNPs) to an oscillating magnetic field outside the linear response region is important for several applications including magnetic hyperthermia, magnetic resonance imaging and biodetection. The size and magnetic moment are two critical parameters for the performance of a colloidal MNP dispersion. We present and demonstrate the use of optomagnetic (OM) and AC susceptibility (ACS) measurements vs. frequency and magnetic field strength to obtain the size and magnetic moment distributions including the correlation between the distributions. The correlation between the size and the magnetic moment contains information on the morphology and intrinsic structure of the particle. In OM measurements, the variation of the second harmonic light transmission through a dispersion of MNPs is measured in response to an oscillating magnetic field. We solve the Fokker-Planck equations for MNPs with a permanent magnetic moment, and develop analytical approximations to the ACS and the OM signals that also account for the change in the curve shapes with increasing field strength. Further, we describe the influence of induced magnetic moments on the signals, by solving the Fokker-Planck equation for particles, which apart from the permanent magnetic moment may also have an induced magnetic moment and shape anisotropy. Using the results from the Fokker-Planck calculations we fit ACS and OM measurements on two multi-core particle systems. The obtained fit parameters also describe the correlations between the magnetic moment and size of the particles. From such an analysis on a commercially available polydisperse multicore particle system with an average particle size of $80 \mathrm{~nm}$, we find that the MNP magnetic moment is proportional to the squareroot of the hydrodynamic size.

\section{Introduction}

The Brownian relaxation dynamics of colloidal magnetic nanoparticle (MNP) dispersions in an oscillating magnetic field is important for a variety of applications including magnetic hyperthermia, magnetic resonance imaging (MRI) ${ }^{1}$, Magnetic Particle

\footnotetext{
a Department of Micro- and Nanotechnology, DTU Nanotech, Bldg. 345B, Technical University of Denmark, DK-2800 Kongens Lyngby, Denmark, Email: jepf@nanotech.dtu.dk, Mikkel.Hansen@nanotech.dtu.dk

${ }^{b}$ Institute of Electrical Measurement and Fundamental Electrical Engineering, Technische Universität Braunschweig, Braunschweig D-38106, Germany

${ }^{c}$ Instituto de Ciencia de Materiales de Madrid, ICMM/CSIC, Sor Juana Ines de la Cruz 3, 28049 Madrid, Spain

${ }^{d}$ Department of Physics, Chalmers University of Technology, Gothenburg 41296, Sweden

$\dagger$ Electronic Supplementary Information (ESI) available: S1: Langevin dynamics; S2: Derivation of Fokker-Planck system of linear equations; S3: Fit of low- $\beta_{0}$ spectra; S4: New ACS approximation; S5: Analytical OM approximation; S6: Fitting OM data in limiting cases; S7: Fitting to bivariate distribution; S8: Fits of data for the NP2 sample; S9: Particle size distributions obtained by TEM; S10: Matlab implementation of functions; S11: Higher harmonics. See DOI: 10.1039/b000000x/
}

Imaging (MPI) ${ }^{2}$ and biodetection assays ${ }^{3}$.

The dynamic properties of an ensemble of identical MNPs in dilute colloidal dispersion depend on the hydrodynamic size and the magnetic moment of the MNPs, which again are related to their morphology and intrinsic structure. Figure 1a defines the terminology and particle compartments of single-core and multicore MNPs.

In both cases, the MNPs have a particle core of diameter $D_{\mathrm{c}}$ and volume $V_{\mathrm{c}}$ that contains the magnetic material either in form of a single core or as several cores that may be loosely or densely packed. We let the diameter and volume of a core be denoted $d_{\mathrm{c}}$ and $v_{\mathrm{c}}$, respectively. For a single-core particle, the core is identical to the particle core, $V_{\mathrm{c}}=v_{\mathrm{c}}$, and the magnetic moment $m$ is expected to be proportional to the volume $V_{\mathrm{c}}$ of the particle core. For multi-core MNPs with many cores with randomly oriented thermally blocked magnetic moments and with weak or no exchange interaction between the cores, the effective particle magnetic moment $m$ is proportional to the square root of the number of cores times the average magnetic moment of a core 


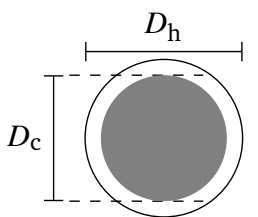

Single-core

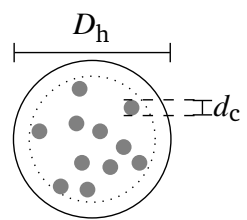

Multi-core
Fig. 1 Schematic of spherical single-core and multi-core particles with hydrodynamic diameter $D_{\mathrm{h}}$ and particle core diameter $D_{\mathrm{c}}$. The diameter of a core (e.g. in a multi-core particle) is denoted $d_{\mathrm{c}}$.

particle ${ }^{4}$. For multi-core MNPs with exchange interacting cores, the magnetic moment scaling will depend on the strength of the exchange interaction.

The MNPs may additionally have a non-magnetic coating and may be functionalised on the surface with molecules. Additionally, when the particles are in a liquid, a layer of liquid may rotate along with the particles and depletion forces may also play a role. All of the above factors contribute to the effective hydrodynamic diameter $D_{\mathrm{h}}$ and volume $V_{\mathrm{h}}$ of a particle that determine the Brownian rotation response of the particle. Specifically, the rotational diffusion with respect to $\theta=0$ is described by the mean-square angular deviation in time $t$ as

$$
\left\langle\theta^{2}\right\rangle=2 D_{\mathrm{r}} t
$$

with the rotational diffusion coefficient

$$
D_{\mathrm{r}}=k_{\mathrm{B}} T / f_{\mathrm{r}}
$$

where $k_{\mathrm{B}} T$ is the thermal energy and $f_{\mathrm{r}}$ is the rotational friction coefficient. For a sphere $f_{\mathrm{r} \text {,sphere }}=\pi \eta D_{\mathrm{h}}^{3}$, where $\eta$ is the viscosity of the liquid. As a result of the Brownian relaxation, the timedependent magnetisation in zero magnetic field of an ensemble of MNPs with an initial magnetisation $M(0)$ can be written as

$$
M(t)=M(0) \exp \left(-t / \tau_{\mathrm{B}}\right)
$$

with the Brownian relaxation time

$$
\tau_{\mathrm{B}}=\frac{1}{2 D_{\mathrm{r}}}=\frac{3 \eta V_{\mathrm{h}}}{k_{\mathrm{B}} T}=\frac{\pi \eta D_{\mathrm{h}}^{3}}{2 k_{\mathrm{B}} T} .
$$

The Brownian relaxation dynamics of a colloidal dispersion of MNPs displaying Brownian relaxation can be studied using AC susceptibility (ACS) measurements by measuring the frequencydependent magnetisation ${ }^{5-13}$. In the linear response regime at low applied magnetic field amplitudes, the dynamic first harmonic magnetic susceptibility of a dilute ensemble of identical MNPs, each having a permanent magnetic moment $m$, can be described using the Debye model

$$
\chi_{1}=\chi_{1}^{\prime}-\mathrm{i} \chi_{1}^{\prime \prime}=\chi_{\infty}+\frac{\chi_{0}-\chi_{\infty}}{1+\mathrm{i} f / f_{\mathrm{B}}}
$$

where $\chi_{0}$ and $\chi_{\infty}$ are the magnetic susceptibilities of the sample at zero and infinite frequencies, respectively, and $f_{\mathrm{B}}$ is the Brownian relaxation frequency given by

$$
f_{\mathrm{B}}=\frac{1}{2 \pi \tau_{\mathrm{B}}}=\frac{k_{\mathrm{B}} T}{\pi^{2} \eta D_{\mathrm{h}}^{3}} .
$$

The Debye model is only applicable in the range of low applied magnetic field amplitudes and the general dynamic fielddependent response has to be calculated numerically. To this purpose, the time evolution of the probability density function for a particle ensemble can efficiently be calculated using the FokkerPlanck equation, and the dynamic magnetisation can be found from the probability density function. Yoshida and Enpuku developed an analytical approximation to the magnetic susceptibility by fitting phenomenological expressions to the increase in the peak position of the imaginary part of the magnetic susceptibility and the ratio between the real and imaginary part at low frequency ${ }^{14}$. A slightly improved expression introduced by Ludwig et al. ${ }^{2}$ was shown to fit the peak position of the imaginary part of the magnetic susceptibility for large applied magnetic field amplitudes ${ }^{15}$. Later Gratz and Tschöpe also proposed an improved ACS approximation by fitting the Debye model to spectra obtained from Fokker-Planck calculations ${ }^{16}$. For all approximations, the curve shape was assumed identical to that of the Debye model. In addition, the approximations only considered MNPs with a permanent magnetic moment.

The modulation of the optical transmission through a colloidal MNP dispersion in response to an applied oscillating magnetic field has also been used to study the nanoparticle dynamics ${ }^{16-20}$. The method was termed optomagnetic (OM) to distinguish it from the traditional magnetooptical effects such as the Kerr and Faraday effects. In OM measurements, as implemented by us ${ }^{20,21}$, the 2 nd harmonic variation of the light intensity transmitted through a colloidal MNP dispersion is measured as a function of the frequency of an oscillating magnetic field applied along the light propagation direction.

We recently developed an analytical model for the low-field OM signal from particles with a permanent magnetic moment using fundamental relations and a few assumptions ${ }^{20}$. The dynamic OM signal at finite fields has also been calculated using the Fokker-Planck equation ${ }^{16}$; however, in that study only the characteristic frequency, defined as the frequency with a phase delay of $45^{\circ}$ relative to the square of the magnetic field signal, was considered.

In this work, we present and demonstrate the use of OM and ACS measurements vs. magnetic field amplitude and frequency to extract the correlation between magnetic moment and size without prior knowledge of the particle structure and morphology. We develop more precise analytical models for the ACS and OM signals for particles with a permanent magnetic moment, and we further describe the influence of an induced magnetic moment on the OM and ACS signals.

In section 2 we solve the Fokker-Planck equation for MNPs, which in addition to a permanent magnetic moment may also have an induced magnetic moment and shape anisotropy. We describe the OM and ACS signals for disperse distributions of particles. In Section 3 we present materials and methods. In Section 
4 we use the results to investigate: (1) the validity of the lowfield OM and ACS models, (2) the field-dependent ACS and OM signals, and (3) the impact of an induced magnetic moment on the signals. For particles with only a permanent magnetic moment, we also develop analytical approximations for the fielddependent dynamic ACS and OM responses, which account for the change in the shape of the curves.

Finally, we report the results of experimental studies on two multi-core MNP systems, NP1 and NP2. Using ACS and OM measurements performed at thirteen magnetic field amplitudes we demonstrate in Section 5 that fits obtained using the solution to the Fokker-Planck equation allow us to obtain the correlation between the magnetic moment and the size of the particles in addition to the number-weighted distributions of $m$ and $V_{\mathrm{h}}$.

\section{Theory}

\subsection{Magnetic nanoparticle in an applied magnetic field}
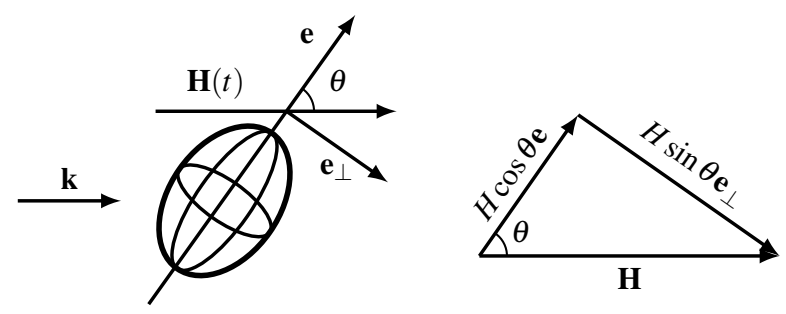

Fig. 2 Schematic of a magnetic nanoparticle in an oscillating magnetic field $\mathbf{H}$ applied in the horizontal direction that also coincides with the light propagation vector $\mathbf{k}$ in optomagnetic measurements. $\theta$ is defined as the angle between the permanent particle magnetic moment $\mathbf{m}=m \mathbf{e}$ and the horizontal direction.

In this work, we will consider an ellipsoidal magnetic nanoparticle, which displays rotational symmetry around a direction e. We will let $D_{\mathrm{c}}$ denote the diameter of the particle core in the direction perpendicular to $\mathbf{e}$ and let the aspect ratio of the particle core (ratio of dimension along $\mathbf{e}$ to that perpendicular to $\mathbf{e}$ ) be denoted $p_{\mathrm{c}}$. The hydrodynamic diameter is defined analogously.

To simplify the treatment of the magnetic behaviour, we will describe the total magnetic moment of a particle as the sum of a permanent magnetic moment and an induced magnetic moment described by a constant, real and homogeneous magnetic susceptibility and the shape of the particle core. This description is representative for a particle containing large thermally blocked magnetic crystals and/or smaller crystals that are superparamagnetic with a Néel relaxation time much lower than the Brownian relaxation time of the particle. This simplified description is not valid for a particle containing cores with Néel relaxation times comparable to the Brownian relaxation time of the particle where the magnetic moment of a particle depends on the frequency of the applied field and has a magnitude and orientation that deviate from a simple superposition of the permanent and induced magnetic moments.

The permanent magnetic moment is assumed to be aligned along e, i.e., $\mathbf{m}=m \mathbf{e}$, and its magnitude and direction relative to the particle is constant and field-independent. The field-induced magnetic moment of the homogeneously magnetised particle core is described in terms of $\chi$ and the demagnetisation factors $N_{\|}$and $N_{\perp}$ along and perpendicular to e, respectively.

Below, we describe the magnetic moment of a particle and the torque on such a particle when placed in a magnetic field as indicated in Fig. 2. The magnetic field $\mathbf{H}$ forms an angle $\theta$ to $\mathbf{e}$ and can be decomposed in terms of $\mathbf{e}$ and $\mathbf{e}_{\perp}$ as $\mathbf{H}=\mathbf{H}_{\|}+\mathbf{H}_{\perp}$ with $\mathbf{H}_{\|}=\mathbf{e}(\mathbf{e} \cdot \mathbf{H})=\mathbf{e} H \cos \theta$ and $\mathbf{H}_{\perp}=\mathbf{H}-H_{\|} \mathbf{e}=\mathbf{e}_{\perp} H \sin \theta$. The vector $\mathbf{k}$ indicates the direction of $\mathbf{H}$ and of the light propagation in the optomagnetic measurements.

With these assumptions and definitions, the total magnetic moment of the particle can be written as

$$
\mathbf{m}_{\mathrm{tot}}=m \mathbf{e}+\chi V_{\mathrm{c}} H\left[\frac{\cos \theta}{1+\chi N_{\|}} \mathbf{e}+\frac{\sin \theta}{1+\chi N_{\perp}} \mathbf{e}_{\perp}\right]
$$

where $V_{\mathrm{c}}=\frac{\pi}{6} D_{\mathrm{c}}^{3} p_{\mathrm{c}}$ is the volume of the particle core. For prolate ellipsoids with aspect ratio $p_{\mathrm{c}}$, the value of $N_{\perp}$ is

$$
N_{\perp}=\frac{1}{2 p_{\mathrm{c}} \xi^{2}}\left[p_{\mathrm{c}}-\frac{1}{p_{\mathrm{c}} \xi} \tanh ^{-1} \xi\right],
$$

with $\xi=\sqrt{p_{\mathrm{c}}^{2}-1} / p_{\mathrm{c}}$ and $N_{\|}=1-2 N_{\perp}{ }^{22}$.

The magnetic moment described in Eq. (7) can be written in the non-orthogonal basis spanned by $\mathbf{H}$ and $\mathbf{e}$ using that $\mathbf{e}_{\perp} H \sin \theta=$ $\mathbf{H}-\mathbf{e} H \cos \theta$ as

$$
\mathbf{m}_{\mathrm{tot}}=m \mathbf{e}+V_{\mathrm{c}}\left(\widetilde{K}(\mathbf{e} \cdot \mathbf{H}) \mathbf{e}+\mathbf{H} \frac{\chi}{1+\chi N_{\perp}}\right)
$$

with the shape anisotropy factor $\widetilde{K}$ given by

$$
\widetilde{K}=\frac{\chi^{2}\left(N_{\perp}-N_{\|}\right)}{\left(1+\chi N_{\|}\right)\left(1+\chi N_{\perp}\right)} .
$$

Note, that for a prolate ellipsoid, the highest value of $\tilde{K}$ is found in the limit $\chi \rightarrow \infty$ where $\tilde{K} \rightarrow\left(N_{\perp}-N_{\|}\right) /\left(N_{\|} \cdot N_{\perp}\right)$.

The magnetic torque $\tau=\mu_{0} \mathbf{m}_{\text {tot }} \times \mathbf{H}$ on the particle is

$$
\tau=\mu_{0} m(\mathbf{e} \times \mathbf{H})+\mu_{0} \widetilde{K} V_{\mathrm{c}}(\mathbf{e} \cdot \mathbf{H})(\mathbf{e} \times \mathbf{H})
$$

where $\mu_{0}$ is the permeability of free space. The first term is the torque generated by the permanent magnetic moment of the particle. The second term is the torque arising from the induced magnetic moment.

\subsection{AC susceptibility and optomagnetic signals}

The rotational dynamics of the particles can be probed in measurements of the magnetisation of a colloidal dispersion of nanoparticles in response to an applied oscillating magnetic field (AC susceptibility measurements) or by corresponding measurements of the modulation of light transmitted through the dispersion (optomagnetic measurements).

The time-dependent magnetisation of a monodisperse dilute ensemble of nanoparticles can be calculated from Eq. (9) as

$$
M(t)=n m \overline{e_{z}}(t)+n V_{\mathrm{c}} H(t)\left[\widetilde{K} \bar{e}_{z}^{2}(t)+\frac{\chi}{1+\chi N_{\perp}}\right],
$$

where $n$ is the particle number concentration and $\overline{e_{z}}(t)$ and $\overline{e_{z}^{2}}(t)$ 
denote the time-dependent ensemble averages of $e_{z}=\cos \theta$ and $e_{z}^{2}=\cos ^{2} \theta$, respectively.

The optomagnetic signal for a monodisperse dilute ensemble of nanoparticles is ${ }^{20}$

$$
\frac{V(t)}{V_{\text {ref }}}=1-n z \Delta \sigma\left(\overline{e_{z}^{2}}(t)-\frac{1}{3}\right),
$$

where $z$ is the light path length, $\Delta \sigma$ is the difference in extinction cross sections for a particle with $\mathbf{e}$ parallel and perpendicular to $\mathbf{k}$, respectively, and $V_{\text {ref }}$ is the photodetector signal for randomly oriented particles (obtained in zero magnetic field).

To find the AC susceptibility (ACS) and optomagnetic (OM) responses, $\overline{e_{z}}(t)$ and $\overline{e_{z}^{2}}(t)$ must be determined. Below we use the Fokker-Planck equation to find the dynamic ensemble averages of $\cos \theta\left(=\overline{e_{z}}\right)$ and $\cos ^{2} \theta\left(=\overline{e_{z}^{2}}\right)$ expressed in terms of their $p^{\prime}$ th harmonics $\overline{e_{z, p}}$ and $\overline{e_{z, p}^{2}}$, respectively.

\subsection{Fokker-Planck equation for the dynamic response}

The Fokker-Planck equation describes the time evolution of the probability distribution of particle orientations, $f(\theta, t)$, in response to an external stimulus. In this case we are only considering the torque on the particle $\tau(\theta, t)$ due to an external magnetic field

$$
H=H_{0} \cos \omega t,
$$

where $\omega=2 \pi f$ is the angular frequency. The Fokker-Planck equation can be written as ${ }^{23}$

$$
\frac{\partial f(\boldsymbol{\theta}, t)}{\partial t}=\frac{1}{2 \tau_{\mathrm{B}}} \boldsymbol{L} \cdot\left[\boldsymbol{L} f(\boldsymbol{\theta}, t)+\frac{1}{k_{\mathrm{B}} T} \tau(\boldsymbol{\theta}, t) f(\boldsymbol{\theta}, t)\right]
$$

where $\boldsymbol{L}=\mathbf{e} \times \nabla_{\mathbf{e}}$ is the rotation operator and $\tau_{\mathrm{B}}$ is given by Eq. (4). Written in terms of $f_{\mathrm{B}}$ (Eq. (6)) and inserting the torque $\tau(\theta, t)$ (Eq. (11)) in the Fokker-Planck equation we obtain in spherical coordinates

$$
\begin{aligned}
\frac{\partial f(\theta, t)}{\partial t}=\frac{\pi f_{\mathrm{B}}}{\sin \theta} \frac{\partial}{\partial \theta}[ & \sin \theta \frac{\partial f(\theta, t)}{\partial \theta}+ \\
& \left.\sin ^{2} \theta\left(\beta_{0} \cos \omega t+\gamma_{0} \cos ^{2} \omega t \cos \theta\right) f(\theta, t)\right]
\end{aligned}
$$

with

$$
\begin{aligned}
& \beta_{0}=\frac{m \mu_{0} H_{0}}{k_{\mathrm{B}} T}=\sqrt{\alpha^{-1} \gamma_{0}} \\
& \gamma_{0}=\frac{\mu_{0} \widetilde{K} V_{\mathrm{c}} H_{0}^{2}}{k_{\mathrm{B}} T}=\alpha \beta_{0}^{2}
\end{aligned}
$$

where

$$
\alpha=\gamma_{0} / \beta_{0}^{2}=\frac{\widetilde{K} V_{\mathrm{c}} k_{\mathrm{B}} T}{\mu_{0} m^{2}} .
$$

The parameter $\alpha$ linking $\gamma_{0}$ and $\beta_{0}$ is a measure of the relative importance of the induced magnetic moment and it depends on the nanoparticle properties and the temperature but not on the applied magnetic field. For $\alpha=0$, the particles have only a per- manent magnetic moment, and for $\alpha^{-1}=0$ the particles have only an induced magnetic moment.

Following ${ }^{23}$, we solve Eq. (16) by expanding the distribution function in harmonics and Legendre polynomials as

$$
f(x, t)=\sum_{l=0}^{\infty} C_{l}(t) P_{l}(x)
$$

with

$$
C_{l}(t)=\sum_{p=0}^{\infty}\left(A_{l, p} \cos p \omega t+B_{l, p} \sin p \omega t\right)
$$

where $B_{l, 0}=0$.

Insertion of Eq. (20) in Eq. (16) results in an infinite system of linear equations in $A_{l, p}$ and $B_{l, p}$ for even values of $l+p$; for details see Eqs. (S15) and (S15) and the derivation in Section S2, $\mathrm{ESI}^{\dagger}$. The equations can be solved numerically (e.g., using the LSQR algorithm) by truncating the sums in Eqs. (20) and (21) at sufficiently high values of $l$ and $p$.

From the solution $f(x, t)$, the ensemble averages $\overline{e_{z}}$ and $\overline{e_{z}^{2}}$ can be calculated using the orthogonality of the Legendre polynomials as

$$
\begin{aligned}
& \overline{e_{z}}(t)=\int_{-1}^{1} x f(x, t) \mathrm{d} x=\frac{2}{3} \sum_{p=0}^{\infty}\left(A_{1, p} \cos p \omega t+B_{1, p} \sin p \omega t\right) \\
& \overline{e_{z}^{2}}(t)=\int_{-1}^{1} x^{2} f(x, t) \mathrm{d} x=\frac{1}{3}+\frac{4}{15} \sum_{p=0}^{\infty}\left(A_{2, p} \cos p \omega t+B_{2, p} \sin p \omega t\right)
\end{aligned}
$$

In Section S2.1, $\mathrm{ESI}^{\dagger}$ we show that the first harmonic of the AC susceptibility, $\chi_{1}=M_{1} / H_{0}$, and the second harmonic of the cosinereferenced optomagnetic signal, $\widetilde{V}_{2} / V_{\text {ref }}$, can be written in terms of the complex Fourier series coefficients as

$$
\begin{aligned}
\chi_{1} & =\frac{n k_{B} T}{\mu_{0} H_{0}^{2}}\left(\beta_{0} \overline{e_{z, 1}}+\frac{\gamma_{0}}{2}\left(\overline{e_{z, 2}^{2}}+2 \overline{e_{z, 0}^{2}}\right)\right)+\frac{n \chi V_{\mathrm{c}}}{1+\chi N_{\perp}} \\
\frac{\widetilde{V}_{2}}{V_{\text {ref }}} & =-n z \Delta \sigma \overline{e_{z, 2}^{2}}
\end{aligned}
$$

where the complex Fourier coefficients are given by

$$
\begin{aligned}
& \overline{e_{z, 1}}=2\left(A_{1,1}+\mathrm{i} B_{1,1}\right) / 3 \\
& \overline{e_{z, 2}^{2}}=4\left(A_{2,2}+\mathrm{i} B_{2,2}\right) / 15 \\
& \overline{e_{z, 0}^{2}}=4 A_{2,0} / 15+1 / 3
\end{aligned}
$$

In the ACS signal, Eq. (24), the first term in the parentheses is due to the permanent magnetic moment and it is proportional to the first harmonic of the ensemble average of $\cos \theta$. The second term is due to the induced magnetic moment and it depends on the second and zeroth harmonics of the ensemble average of $\cos ^{2} \theta$. Thus, the ACS signal from the induced moment is linked to the optomagnetic signal, Eq. (25), which also depends on $\cos ^{2} \theta$.

To test the validity of the above Fokker-Planck derivation, we also performed stochastic dynamic simulations (Section S1, ESI ${ }^{\dagger}$ ). The results of the stochastic simulations were found to be in per- 
fect agreement with those from the Fokker-Planck simulations (Section S1.2, $\mathrm{ESI}^{\dagger}$ ).

\subsection{Disperse distributions}

\section{General expressions.}

The solution of the Fokker-Planck equation system, Eqs. (S15) and (S16), only depends on three parameters: $f / f_{\mathrm{B}}, \beta_{0}$ and $\gamma_{0}$. Consequently, $\overline{e_{z}}$ and $\overline{e_{z}^{2}}$ also only depend on these parameters. Further, the parameters can be described by the three materialdependent parameters $m, \widetilde{K} V_{\mathrm{c}}$, and $f_{\mathrm{B}}$ together with $T, H_{0}$ and $f$, which are controlled in the experiment. Generally, we can write the ACS signal as

$$
\begin{array}{r}
\chi_{1}=\frac{n k_{\mathrm{B}} T}{\mu_{0} H_{0}^{2}} \iiint\left[\frac{\gamma_{0}}{2}\left(\overline{e_{z, 2}^{2}}+2 \overline{e_{z, 0}^{2}}\right)+\beta_{0} \overline{e_{z, 1}}\right] \\
\times p\left(f_{\mathrm{B}}, m, \widetilde{K} V_{\mathrm{c}}\right) \mathrm{d} f_{\mathrm{B}} \mathrm{d} m \mathrm{~d}\left(\widetilde{K} V_{\mathrm{c}}\right)+\chi_{\infty},
\end{array}
$$

where $p\left(f_{\mathrm{B}}, m, \widetilde{K} V_{\mathrm{c}}\right)$ denotes the number fraction of particles with Brownian relaxation frequencies between $f_{\mathrm{B}}$ and $f_{\mathrm{B}}+\mathrm{d} f_{\mathrm{B}}$, permanent magnetic moments between $m$ and $m+\mathrm{d} m$, and $\widetilde{K} V_{\mathrm{c}}$-values between $\widetilde{K} V_{\mathrm{c}}$ and $\widetilde{K} V_{\mathrm{c}}+\mathrm{d}\left(\widetilde{K} V_{\mathrm{c}}\right)$. In Eq. (29), $\chi_{\infty}=$ $n \int \frac{\chi V_{\mathrm{c}}}{1+\chi N_{\perp}} p\left(V_{\mathrm{c}}\right) \mathrm{d} V_{\mathrm{c}}$ where $p\left(V_{\mathrm{c}}\right)$ is the number fraction of particles with core volumes between $V_{\mathrm{c}}$ and $V_{\mathrm{c}}+\mathrm{d} V_{\mathrm{c}}$.

The OM signal can generally be written as

$$
\frac{\widetilde{V}_{2}}{V_{\text {ref }}}=-n z \iiint \Delta \sigma \overline{e_{z, 2}^{2}} p\left(f_{\mathrm{B}}, m, \widetilde{K} V_{\mathrm{c}}\right) \mathrm{d} f_{\mathrm{B}} \mathrm{d} m \mathrm{~d}\left(\widetilde{K} V_{\mathrm{c}}\right) .
$$

The magnetic susceptibility signal $\chi_{1}$ depends on $m, \widetilde{K} V_{\mathrm{c}}$ and $f_{\mathrm{B}}$, whereas the optomagnetic signal has an additional $\Delta \sigma$-weighting of the signal. Information on the particle structure and morphology is needed to obtain the correlation between $m, \widetilde{K} V_{\mathrm{c}}, f_{\mathrm{B}}$ and $\Delta \sigma$. Below, we make simplifying assumptions concerning the correlation between these parameters.

\section{Parameter correlations.}

For particles with aspect ratios close to unity, we can assume that the particle properties $m, f_{\mathrm{B}}$ and $\Delta \sigma$ are independent of the shape of the particles ${ }^{20}$. Further, we assume that the parameters are related to the particle core volume $V_{\mathrm{c}}$ according to power law relations as

$$
\begin{gathered}
f_{\mathrm{B}}=\widetilde{f}_{\mathrm{B}}\left(\frac{V_{\mathrm{c}}}{\widetilde{V}_{\mathrm{c}}}\right)^{-n_{\mathrm{h}}} \\
m=\widetilde{m}\left(\frac{V_{\mathrm{c}}}{\widetilde{V}_{\mathrm{c}}}\right)^{n_{m}} \\
\Delta \sigma=\widetilde{\Delta \sigma}\left(\frac{V_{\mathrm{c}}}{\widetilde{V}_{\mathrm{c}}}\right)^{n_{\sigma}}
\end{gathered}
$$

Here, $\widetilde{f}_{\mathrm{B}}, \widetilde{m}$ and $\widetilde{\Delta \sigma}$ are the Brownian relaxation frequency, the permanent magnetic moment and the extinction cross section difference when $V_{\mathrm{c}}=\widetilde{V}_{\mathrm{c}}$. The exponents, $n_{\mathrm{h}}, n_{m}$ and $n_{\sigma}$ depend on the morphology and internal structure of the particles as discussed further below. Note, that the above expressions also allow for the parameters to be uncorrelated in which case the correspon- ding exponent is zero.

For particles with a thin non-magnetic shell the particle core size is proportional to the hydrodynamic size (and hence also the Brownian relaxation frequency) whereby $n_{\mathrm{h}}=1$. If the nonmagnetic shell is thick and of varying thickness, we anticipate that the two parameters are not correlated, i.e., $n_{\mathrm{h}}=0$.

The extinction cross sections and hence $\Delta \sigma$ scale with the extinction properties of the particles. Since the refractive indices of the surrounding water and of the non-magnetic shell are typically similar but smaller than the refractive index of the particle core, the extinction properties of the particles are mainly determined by the optical properties of the particle core. If absorption dominates, the extinction is proportional to the volume of the particle core $\left(n_{\sigma}=1\right)$.

Finally, the permanent magnetic moment scales with the amount of magnetic material and therefore with the particle core volume. For single-core particles, the magnetic moment is proportional to the particle core volume, $m=M_{\mathrm{s}} V_{\mathrm{c}}$, where $M_{\mathrm{S}}$ is the magnetisation of the core material $\left(n_{m}=1\right)$. For multi-core particles with many randomly oriented thermally blocked core magnetic moments, the particle magnetic moment is expected to be proportional to the square root of the particle core volume $\left(n_{m}=0.5\right)^{4}$. In more detail, considering such a particle as consisting of $N$ cores of identical size filling up a fraction $\xi$ of the particle core volume, we can estimate $N$ as $N=\xi V_{\mathrm{c}} / v_{\mathrm{c}}$. Moreover, in the limit of large $N$, we can write the permanent particle magnetic moment as $m \approx N^{1 / 2} M_{\mathrm{s}} v_{\mathrm{c}}=\left(\xi V_{\mathrm{c}} v_{\mathrm{c}}\right)^{1 / 2} M_{\mathrm{s}}$ corresponding to $n_{m}=0.5$ and $\tilde{m} / \tilde{V}_{\mathrm{c}}^{1 / 2}=\left(\xi v_{\mathrm{c}}\right)^{1 / 2} M_{\mathrm{s}}$.

Using the parameter correlations, Eqs. (32) and (33), we observe that $m$ and $\Delta \sigma$ are correlated according to

$$
\Delta \sigma=\widetilde{\Delta \sigma}\left(\frac{m}{\widetilde{m}}\right)^{n_{\sigma} / n_{m}}
$$

where a particle with permanent magnetic moment $\widetilde{m}$ has an extinction cross section difference of $\widetilde{\Delta \sigma}$.

For a constant value of $\tilde{K}$, the general three-dimensional distribution, $p\left(f_{\mathrm{B}}, m, \widetilde{K} V_{\mathrm{c}}\right)$, in Eqs. (29) and (30) can be reduced to a two-dimensional distribution $p\left(f_{\mathrm{B}}, m\right)$ in special cases. Common for these is that

$$
\chi_{1}=\frac{n k_{\mathrm{B}} T}{\mu_{0} H_{0}^{2}} \iint\left[\frac{\gamma_{0}}{2}\left(\overline{e_{z, 2}^{2}}+2 \overline{e_{z, 0}^{2}}\right)+\beta_{0} \overline{e_{z, 1}}\right] p\left(f_{\mathrm{B}}, m\right) \mathrm{d} f_{\mathrm{B}} \mathrm{d} m+\chi_{\infty}
$$

$$
\frac{\widetilde{V_{2}}}{V_{\text {ref }}} \simeq-n z \frac{\widetilde{\Delta \sigma}}{\widetilde{m}^{n_{\sigma} / n_{m}}} \iint m^{n_{\sigma} / n_{m}} \overline{e_{z, 2}^{2}} p\left(f_{\mathrm{B}}, m\right) \mathrm{d} f_{\mathrm{B}} \mathrm{d} m
$$

where the relation between $\gamma_{0}$ and $\beta_{0}$ has to be specified. Note, that the complex Fourier coefficients, $\overline{e_{z, 1}^{1}}, \overline{e_{z, 0}^{2}}$ and $\overline{e_{z, 2}^{2}}$, in the above expressions all depend on $\beta_{0}, \gamma_{0}$, and $f_{\mathrm{B}}$ and moreover that $\gamma_{0} \propto \tilde{K}$ and $\beta_{0} \propto m$. However, if $\gamma_{0}$ can be written as a function of $\beta_{0}$, the above expressions are unique and well-defined.

The simplest case is when the shape anisotropy is negligible $\left(\tilde{K}=\gamma_{0}=0\right)$, where Eq. (35) reduces to

$$
\chi_{1}=\frac{n}{H_{0}} \iint m \overline{e_{z, 1}} p\left(f_{\mathrm{B}}, m\right) \mathrm{d} f_{\mathrm{B}} \mathrm{d} m+\chi_{\infty} .
$$


The form of Eq. (36) for the optomagnetic signal is unchanged for $\tilde{K}=0$.

When $\tilde{K} \neq 0$, we need to make additional assumptions to reduce the distribution function to two dimensions by making a unique link between $\gamma_{0}$ and $\beta_{0}$ in Eq. (35). We will consider the two cases of $n_{m}=1$ and $n_{m}=0.5$ corresponding to the single-core and multicore particles as described above.

For the single-core particles $\left(n_{m}=1\right)$, we insert $m=M_{\mathrm{s}} V_{\mathrm{c}}$ in the definitions of $\alpha, \beta_{0}$ and $\gamma_{0}$ and find

$$
\gamma_{0}^{\mathrm{sc}}=\alpha \beta_{0}^{2}=H_{0} \frac{\widetilde{K}}{M_{\mathrm{s}}} \beta_{0} \quad\left(\text { single-core particles with } n_{m}=1\right) .
$$

Thus, for such particles $\alpha \beta_{0}$ is a constant that depends on $H_{0}, \tilde{K}$ and $M_{\mathrm{s}}$, but not the volume of the particle core.

For the described multi-core particles $\left(n_{m}=0.5\right)$, we insert $m=$ $\left(\xi V_{\mathrm{c}} v_{\mathrm{c}}\right)^{1 / 2} M_{\mathrm{s}}$ in the definition of $\alpha$ and find

$\alpha^{\mathrm{mc}}=\gamma_{0}^{\mathrm{mc}} / \beta_{0}^{2}=\frac{\widetilde{K} k_{\mathrm{B}} T}{\mu_{0} \xi v_{\mathrm{c}} M_{\mathrm{s}}^{2}} \quad$ (multi-core particles with $\left.n_{m}=0.5\right)$.

Thus, for such multi-core particles at a fixed temperature, $\alpha=$ $\alpha^{\mathrm{mc}}$ is a constant that depends on the material, the packing fraction of the cores, of the volume of the cores and on the shape of the particle but not on the size of the particle core.

Below, we consider the case of monomodal lognormal distributions of $f_{\mathrm{B}}$ and $m$ corresponding to any of the above considered cases, where we furthermore allow $f_{\mathrm{B}}$ and $m$ to be correlated. This can be described using a bivariate lognormal distribution.

\section{Bivariate lognormal distribution.}

The two-dimensional bivariate lognormal distribution of $f_{\mathrm{B}}$ and $m$ is given as

$p\left(f_{\mathrm{B}}, m\right) \mathrm{d} f_{\mathrm{B}} \mathrm{d} m=\frac{\exp \left(\frac{-1}{2\left(1-\rho^{2}\right)}\left[\frac{\ln ^{2} \frac{f_{\mathrm{B}}}{\mu_{\mu_{\mathrm{B}}}}}{\sigma_{f_{\mathrm{B}}}^{2}}+\frac{\ln ^{2} \frac{m}{\mu_{m}}}{\sigma_{m}^{2}}-\frac{2 \rho \ln \frac{f_{\mathrm{B}}}{\mu_{\mathrm{B}_{\mathrm{B}}}} \ln \frac{m}{\mu_{m}}}{\sigma_{f_{\mathrm{B}}} \sigma_{m}}\right]\right)}{2 \pi f_{\mathrm{B}} m \sigma_{f_{\mathrm{B}}} \sigma_{m} \sqrt{1-\rho^{2}}} \mathrm{~d} f_{\mathrm{B}} \mathrm{d} m$

and is described by five parameters: the median permanent magnetic moment, $\mu_{m}$, the median Brownian relaxation frequency, $\mu_{f_{\mathrm{B}}}$, the lognormal widths $\sigma_{m}$ and $\sigma_{f_{\mathrm{B}}}$ and the correlation term $\rho$. For $\rho=0$, the magnetic moments and the Brownian relaxation frequencies are uncorrelated, whereas for $|\rho|=1$ the magnetic moments have a one-to-one correlation to the Brownian relaxation frequencies, meaning that each value of the magnetic moment corresponds to a unique value of the hydrodynamic size. For a bivariate normal distribution, a non-zero $\rho$ value results in the variables being linearly dependent on each other. For a bivariate $\log$-normal distribution, the correlation corresponds to a power law as

$$
m=\widetilde{m}\left(\frac{f_{\mathrm{B}}}{\widetilde{f}_{\mathrm{B}}}\right)^{n_{f_{\mathrm{B}}}}
$$

where a particle has the magnetic moment $\widetilde{m}$ when the Brownian relaxation frequency is $\tilde{f}_{\mathrm{B}}$. The value of $n_{f_{\mathrm{B}}}$ can be calculated from the bivariate fit as

$$
n_{f_{\mathrm{B}}}=\frac{2 \sigma_{f_{\mathrm{B}}} \sigma_{m} \rho}{\sigma_{f_{\mathrm{B}}}^{2}-\sigma_{m}^{2}+\sqrt{\left(\sigma_{f_{\mathrm{B}}}^{2}-\sigma_{m}^{2}\right)^{2}+4 \sigma_{f_{\mathrm{B}}}^{2} \sigma_{m}^{2} \rho^{2}}}
$$

which reduces to $n_{f_{\mathrm{B}}}=\operatorname{sgn}(\rho) \sigma_{m} / \sigma_{f_{\mathrm{B}}}$ for $|\rho|=1$. Combining Eqs. (31), (32) and (41), we also observe that $n_{f_{\mathrm{B}}}=-n_{m} / n_{\mathrm{h}}$. Thus, a fit of the bivariate distribution to a set of data can be used to estimate $n_{f_{\mathrm{B}}}=-n_{m} / n_{\mathrm{h}}$. Further using (or assuming) a relation between $V_{\mathrm{c}}$ and $V_{\mathrm{h}}$, e.g., if the non-magnetic coating on a particle is thin compared to the particle diameter, the value of the magnetic moment exponent $n_{m}$ can be estimated. For the simple case of $n_{\mathrm{h}}=1$, we have $n_{m}=-n_{f_{\mathrm{B}}}$.

\section{Materials and methods}

\subsection{Fokker-Planck simulations}

The Fokker-Planck calculation was implemented in Matlab (Section S10, $\mathrm{ESI}^{\dagger}$ ). Calculations with $\beta_{0}<100$ and $\gamma_{0}=0$ were performed by truncating the linear equations at $l=60$ and $p=60$. Higher values did not change the result. In calculations with $500>\gamma_{0}>0$, the linear equations were truncated at $l=110$ and $p=150$. The convergence of the solution was verified. For large values of $\beta_{0}$ and $\gamma_{0}$ the results did not converge and consequently those results were not used.

\subsection{Magnetic nanoparticle systems}

Two magnetic nanoparticle systems, labeled NP1 and NP2, were studied.

The first system (NP1) was a commercially available multi-core MNP system where the particles had an average diameter of 80 nm (BNF-Dextran, 84-00-801, Micromod, DE). The particle dispersion was diluted to particle concentrations of $0.2 \mathrm{~g} / \mathrm{L}$ in 50 $\mathrm{mM}$ phosphate buffer ( $\mathrm{pH} 8.5$ ) with $0.5 \%$ Tween-20.

The second system (NP2) was produced as part of the EU Nanomag project ${ }^{24}$ with the internal label CSIC-04 in the project. In this sample, the MNPs consisted of dextran-coated $60 \mathrm{~nm}$ multicore particles formed from $30 \mathrm{~nm}$ octahedral-like maghemite nanocrystals. The nanocrystals were synthesized by a modified oxidative precipitation ${ }^{25}$ followed by an oxidative acid treatment ${ }^{26}$, coated with dextran under high-pressure homogenisation conditions $^{27}$ and size sorted using a Sepmag-Q100 magnetic separation system. NP2 was diluted in de-ionized water to a particle concentration of $0.6 \mathrm{~g} / \mathrm{L}$.

The MNP volume fractions in both samples used in this study are low $\left(\xi<10^{-4}\right)$ and we can therefore safely neglect the influence of inter-particle interactions. ${ }^{28}$

\subsection{AC susceptibility measurements}

Measurements of the AC susceptibility were performed at the Technical University of Braunschweig with a setup that was originally designed for measurements of the MNP dynamics in rotating magnetic fields ${ }^{29}$. It consisted of two orthogonal pairs of Helmholtz coils. The stray field from the MNP samples is detected with a differential fluxgate arrangement. In order to perform ACS measurements for different AC field amplitudes, only one of the 
Helmholtz coils was driven with a sine excitation current. Magnetic field amplitudes up to $9 \mathrm{mT}$ could be realized. Spectra were recorded at thirteen different magnetic field amplitudes $\left(\mu_{0} H_{0}\right.$ $[\mathrm{mT}]=0.25,0.5,0.75,1,2,2.5,3,3.5,4,4.5,5,5.5$, and 6). The frequency was swept from $9 \mathrm{~Hz}$ to $5 \mathrm{kHz}$ in 29 logarithmically equidistant steps. At each frequency and AC field amplitude, five averages were taken to produce the measurement value and its standard deviation. For each field strength the average standard deviation for all frequencies was calculated and used in the fitting procedure. The measurements were performed at $25^{\circ} \mathrm{C}$.

\subsection{Optomagnetic measurements}

Optomagnetic measurements were performed at the Technical University of Denmark using a previously described setup ${ }^{21}$. In brief, a laser (Sanyo Blu-ray optical pick-up, $\lambda=780 \mathrm{~nm}$ ) sent light through a measurement chamber on a microfluidic disk. The transmitted light was collected using a photodetector (ThorLabs PDA36A), and the resulting voltage time traces were measured by a data acquisition card (National Instruments 6251), and analysed in Labview using fast Fourier transformation (FFT).

Two electromagnets positioned symmetrically on both sides of the measurement chamber produced a homogeneous sinusoidally varying magnetic field. The magnets were controlled by the data acquisition card via a voltage-controlled current amplifier. The magnetic field strength was calibrated and the phase lag of the magnetic field relative to the applied voltage was measured using a Hall sensor (SS94A2D, Honeywell). The phase of the OM measurements was corrected to be relative to the magnetic excitation. Spectra were recorded at thirteen different field amplitudes $\left(\mu_{0} H_{0}\right.$ $[\mathrm{mT}]=0.33,0.66,0.99,1.33,1.99,2.66,3.31,3.97,4.64,5.30$, $5.96,6.62$, and 7.28). The field strength amplitude was constant within $1 \%$ throughout the entire frequency range. The frequency was swept from $9 \mathrm{~Hz}$ to $1330 \mathrm{~Hz}$ in 17 logarithmically equidistant steps. Each frequency spectrum was recorded in less than 5 min. The uncertainties were estimated from five repeated measurements as the standard deviation on the average on the mean of these measurements at each field strength. The measurements were performed at $22{ }^{\circ} \mathrm{C}$.

The spectra were recorded using a sine magnetic field as the reference signal. The corresponding second harmonic signal is denoted $V_{2}=V_{2}^{\prime}+\mathrm{i} V_{2}^{\prime \prime}$. The relation to the signal obtained using a cosine reference signal, $\widetilde{V}_{2}=\widetilde{V}_{2}^{\prime}-i \widetilde{V}_{2}^{\prime \prime}$, as used in the theory section, is $V_{2}=-i \widetilde{V}_{2}{ }^{20}$, i.e.,

$$
\begin{aligned}
& \widetilde{V}_{2}^{\prime}=-V_{2}^{\prime \prime} \\
& \widetilde{V}_{2}^{\prime \prime}=-V_{2}^{\prime}
\end{aligned}
$$

To ease the comparison to previous studies, we will present calculated and measured optomagnetic signals below in terms of $V_{2}$, i.e., the values obtained for a sine reference signal.

\subsection{Transmission electron microscopy (TEM)}

A FEI Titan 80-300 TEM equipped with field emission gun and operated at $300 \mathrm{kV}$ at Chalmers University of Technology was used in this study. TEM specimens were prepared by drying a few drops of the nanoparticle dispersion on holey carbon film coated $\mathrm{Cu}$ TEM grids at room temperature. TEM bright field (BF) images of the particles were used to estimate the sizes of the particle cores and of the cores forming the multi-core particles, respectively. The size of each particle was defined as the diameter of the circle enclosing the particle. Thus, the longest dimension of each particle was taken as the size of it. The number of cores in each multi-core particle was directly counted from TEM BF images.

\section{Simulation results}

We first present results of the Fokker-Planck simulations for particles with a negligible induced magnetic moment $\left(\gamma_{0}=\alpha=0\right)$. These results are divided into the low-field behaviour vs. frequency where we compare to a previously presented simplified analytical model and the general behaviour vs. amplitude of the magnetic field, where we present an improved analytical approximation to the AC susceptibility signal as well as an analytical approximation to the optomagnetic signal. Subsequently, we investigate the effect of a non-negligible induced magnetic moment and consider the range of particle properties for which the induced magnetic moment contribution is relevant.

\subsection{Low-field behaviour $\left(\beta_{0} \ll 1, \gamma_{0}=\alpha=0\right.$ )}

First, the validity of the Debye model for ACS data and the analytical model for OM data presented in ${ }^{20}$ was investigated using the results obtained from the Fokker-Planck calculation at low magnetic fields for particles with only a permanent magnetic moment. The calculations were performed with $\beta_{0}=10^{-3}$ and $\gamma_{0}=0$. It was verified that other (lower) values of $\beta_{0}$ produced the same results.

For the AC susceptibility, the Debye model was found to match the Fokker-Planck results with residuals going towards zero for $\beta_{0}$ going to zero (Section S3, ESI ${ }^{\dagger}$ ). Thus, a perfect agreement between the two descriptions was found. For the OM results, a fit of the previously presented analytical model to the low-field OM data obtained from the solution to the Fokker-Planck equation resulted in a Brownian relaxation frequency that was $21 \%$ too large, and with residuals indicating a systematic error on a level of about $1 \%$ (Section S3, $\mathrm{ESI}^{\dagger}$ ). The systematic error was not reduced by lowering $\beta_{0}$ further. Thus, although the analytical model provides a good fit to the OM data, it has to be corrected to be quantitatively correct. We found that the low-field OM signal could be quantitatively described by introducing a correction of the Brownian relaxation frequency, such that

$$
V_{2} \propto \mathrm{i}\left(\frac{1}{1+\mathrm{i} \frac{f}{1.21 f_{\mathrm{B}}}}\right)^{2} .
$$

In the development of the low-field OM model in ${ }^{20}$ it was assumed that the equilibrium relation between $\overline{e_{z}}$ and $\overline{e_{z}^{2}}$ was also valid in non-equilibrium. The Fokker-Planck results show that this assumption was not quantitatively correct but also that the main difference is a $21 \%$ correction of the value of $f_{\mathrm{B}}$ as indicated above. This implies that values of $V_{\mathrm{h}}$ and $D_{\mathrm{h}}$ obtained without the correction underestimate the correct values by $17 \%$ and $6 \%$, respectively. 


\subsection{Field-dependent behaviour and analytical approximati- ons $\left(\gamma_{0}=\alpha=0\right)$}

Having described the low-field case, $\beta_{0} \ll 1$, we now turn to the field dependence of the signals for particles that still only have a permanent magnetic moment. The solid lines in Fig. 3a-d show the Fokker-Planck calculated ACS and OM signals for particles with no induced magnetic moments $\left(\alpha=\gamma_{0}=0\right)$ vs. frequency for a range of values of $\beta_{0}$ corresponding to increasing values of the magnetic field. The signal is normalised to the low-frequency signal amplitude. For increasing values of $\beta_{0}$, the positions of the peaks in $V_{2}^{\prime}$ and $\chi_{1}^{\prime \prime}$ are observed to shift to higher frequencies. The dependence of the peak positions on $\beta_{0}$ is shown in Figs. 3e,g. The shifts of the peaks are accompanied by a general change of the curve shapes where the peaks become increasingly asymmetric for large $\beta_{0}$ values. Likewise, it is observed that the inflection point of $\chi_{1}^{\prime}$ and the frequency where $V_{2}^{\prime \prime}$ changes sign move to higher frequencies. The shape of $V_{2}^{\prime \prime}$ changes dramatically whereas that of $\chi_{1}^{\prime}$ only changes little.

Yoshida and Enpuku have made analytical approximations to the Fokker-Planck results for the AC susceptibility, $\chi_{1}^{2,14}$. They described the field dependence using a parameter for the change of the peak position of $\chi_{1}^{\prime \prime}$ and assumed that the inflection point of $\chi_{1}^{\prime}$ would follow the same dependence. Later, Gratz and Tschöpe followed the same approach but used the peak position found from fits of a Debye model to the spectra ${ }^{16}$. None of the above approximations account for the change of the curve shape. Consequently, they only describe the curves in the regime of weak magnetic fields $\left(\beta_{0}<10\right.$, see Fig. S4, $\left.\mathrm{ESI}^{\dagger}\right)$. Introducing three additional parameters to the description of the curves, we propose a parametric description of the AC susceptibility according to

$$
\begin{aligned}
& \chi_{1}^{\prime}=\chi_{1}(0) \frac{1}{\left(1+\left(f / f_{\mathrm{B} 1}\right)^{2}\right)^{a}} \\
& \chi_{1}^{\prime \prime}=\chi_{1}(0) \frac{\left(c f / f_{\mathrm{B} 2}\right)^{b}}{1+\left(f / f_{\mathrm{B} 2}\right)^{2}}
\end{aligned}
$$

with

$$
\begin{aligned}
f_{\mathrm{B} 1} & =f_{\mathrm{B}}\left(1+0.144 \beta_{0}{ }^{2}\right)^{0.395} \\
f_{\mathrm{B} 2} & =f_{\mathrm{B}}\left(1+0.0908 \beta_{0}{ }^{2}\right)^{0.48} \\
\chi_{1}(0) & =\chi_{0}\left(1-0.0247 \beta_{0}+0.135 \beta_{0}{ }^{1.7}+0.0271 \beta_{0}{ }^{2.7}\right)^{-0.37} \\
a & =1+\frac{0.0252 \beta_{0}{ }^{2.2}}{1+0.239 \beta_{0}{ }^{1.4}+0.0144 \beta_{0}{ }^{2.3}} \\
b & =1-\frac{0.00214 \beta_{0}{ }^{2.5}}{1+0.0737 \beta_{0}{ }^{1.45}+0.00679 \beta_{0}{ }^{2.45}} \\
c & =1+\frac{0.0201 \beta_{0}{ }^{2.2}}{1+0.16 \beta_{0}{ }^{1.4}+0.0229 \beta_{0}{ }^{2.1}}
\end{aligned}
$$

where $\chi_{0}=n m^{2} \mu_{0} /\left(3 k_{\mathrm{B}} T\right)$. In Fig. S5, $\mathrm{ESI}^{\dagger}$ we show the this phenomenological expression accounts for both the peak position and the shape of the AC susceptibility data for $\beta_{0}<300$. The Matlab code for the susceptibility is given in Section S10.3, ESI ${ }^{\dagger}$.

An analytical approximation for the optomagnetic signal was also developed and is reported in Section S5, ESI ${ }^{\dagger}$. However, to describe the complex change of the curve shape, we needed to introduce a total of thirteen parameters - five more than used to describe the magnetic susceptibility signal.

The dotted lines in Fig. 3 show the improved analytical approximations for the ACS and OM signals. The analytical approximations describe the curves with some minor derivations. For the ACS approximation, the deviation is mainly visible for the highest $\beta_{0}$ values and it is found to be below $4 \%$ relative to the maximum amplitude for $\beta_{0}<30$, and below $5 \%$ for $\beta_{0}<100$. The approximation developed by Yoshida and Enpuku ${ }^{2}$ was shown to describe the peak position of $\chi_{1}^{\prime \prime}$ for $\beta_{0}$-values up to $100^{15}$; however, their approximation showed a maximum deviation of the curves from the Fokker-Planck results that was about $10 \%$ for $\beta_{0}=10$, and up to $50 \%$ for $\beta_{0}=100$. If this approximation is used to fit the entire curve rather than only the peak position, such large deviations between the approximation and exact simulations can produce significant errors in the fitting result.

\subsection{Effect of induced magnetic moment $\left(\gamma_{0} \neq 0\right)$}

When an induced magnetic moment is included, anisotropic particles will be affected by a magnetic torque $\left(\gamma_{0} \neq 0\right)$ and the frequency-dependent OM and ACS signals may change.

Fig. 4 shows the signal for $\alpha=0.1$, corresponding to $\gamma_{0}=0.1 \beta_{0}^{2}$. The variation of $\beta_{0}$ is equivalent to changes of the magnetic field strength, $H_{0}$, at fixed temperature for a monodisperse colloidal dispersion of particles with $\alpha=0.1$. For low values of $\beta_{0}$, the curves are similar to those obtained for $\alpha=0$ (blue curves). However, for larger values of $\beta_{0}$, we observe a significant influence of the torque due to the induced magnetic moment. Specifically, it is observed for large values of $\beta_{0}$ that the peak positions of $V_{2}^{\prime}$ and $\chi_{1}^{\prime \prime}$ no longer show a simple increase with $\beta_{0}$ and that the curve shape is significantly deformed.

Figs. 3e,g show the peak positions of $V_{2}^{\prime}$ and $\chi_{1}^{\prime \prime}$ as function of $\beta_{0}$ for the indicated values of $\alpha$. The peak positions are not affected by a small induced magnetic moment ( $\alpha=0.01$ and $\beta_{0}<30$ ). For larger values of $\alpha$, it is observed that the peak positions of $V_{2}^{\prime}$ and $\chi_{1}^{\prime \prime}$ no longer increase monotoneously with $\beta_{0}$ and that $\beta_{0}$-values above an $\alpha$-dependent threshold result in a negative shift of the peak positions. In this regime, the torque due to the induced magnetic moment will dominate over that due to the permanent magnetic moment.

At low values of $\alpha$, an increasing value of $\beta_{0}$ will increase the torque from the permanent magnetic moment and accelerate the rotation of the particles leading to an increase of the apparent Brownian relaxation frequency. However, for $\alpha>0$ and a significant value of $\beta_{0}$, the induced magnetic moment will work against rotation of the particles by more than $90^{\circ}$ as this contribution has two equivalent low-energy orientations separated by $180^{\circ}$. For large values of $\beta_{0}$, this energy contribution will dominate over that due to the permanent magnetic moment and flipping of the particle orientation will be prevented. When the induced mag- 

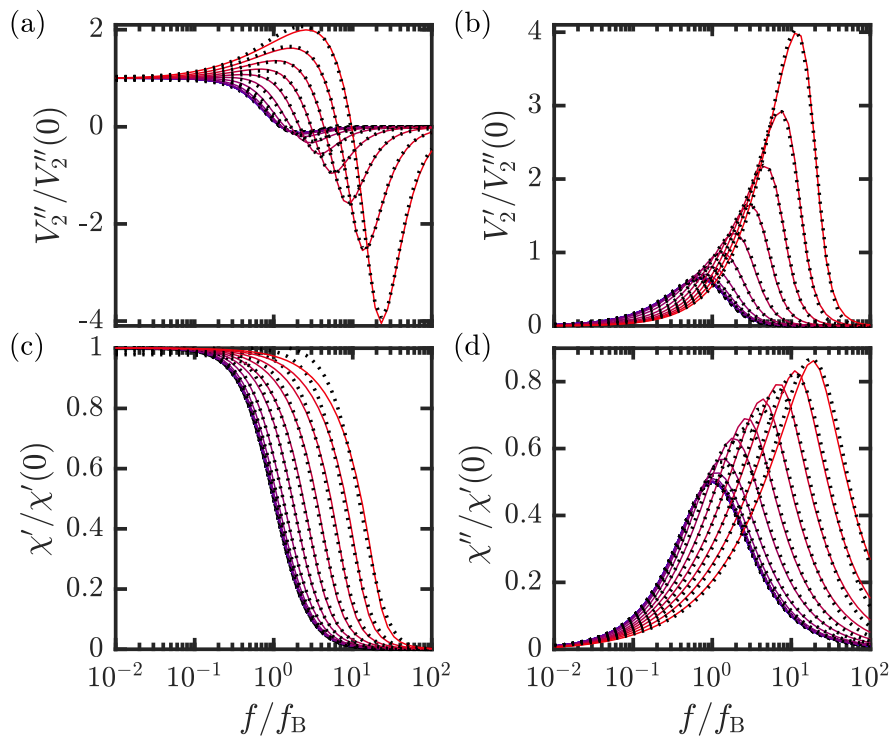
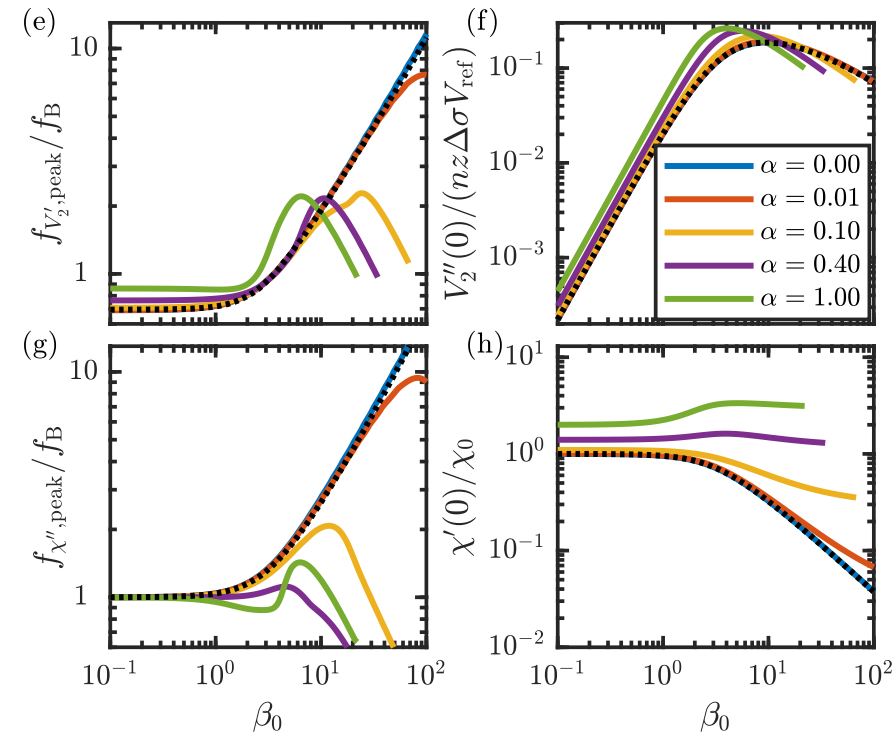

Fig. 3 (a)-(b) Optomagnetic data and (c)-(d) magnetic susceptibility data obtained from Fokker-Planck calculations (solid lines) with $\gamma_{0}=\alpha=0$ for $\beta_{0}$-values ranging from 0.01 (blue) to 100 (red) in 17 logarithmically equidistant steps. Panels (e) and (g) show the peak position dependence on $\beta_{0}$ for the optomagnetic $\left(V_{2}^{\prime}\right)$ and magnetic $\left(\chi_{1}^{\prime \prime}\right)$ signals, respectively, for the $\alpha$-values shown in the legend in (f). (f) The low-frequency amplitudes of the optomagnetic signal $\left(V_{2}^{\prime \prime}\right)$ normalised by $V_{2}^{\prime \prime}(0)=-n z \Delta \sigma V_{\text {ref }}$ and $(\mathrm{h})$ the low-frequency amplitudes of the magnetic signal $\left(\chi_{1}^{\prime \prime}\right)$ normalised by $\chi_{0}$. The dotted lines are the analytical approximations for $\alpha=0$. For (e)-(h) the calculations were performed using the $\alpha$ values shown in the legend of (f). All calculations were performed with $\chi_{\infty}=0$.

netic moment dominates the signal, the frequency dependence of the ACS signal originates from $\overline{e_{z, 2}^{2}}$ and $\overline{e_{z, 0}^{2}}$ as can be seen from Eq. (24). Therefore, for large values of $\alpha$ and $\beta_{0}$, the magnetic susceptibility signal will resemble the OM signal.

Figs. 3f,h show the low-frequency magnitudes of the OM and ACS signals vs. $\beta_{0}$ for the indicated values of $\alpha$. A small induced magnetic moment ( $\alpha=0.01$ ) is not observable in the magnitude of the OM signal. However, for $\beta_{0}>10$ an increase is observed in the low-frequency ACS signal (Fig. 3h). This increase is primarily caused by the increase of the induced magnetic moment and not by an increase in $\overline{e_{z, 1}}$. From the OM signal for $\alpha=0.01$, we observe that $\overline{e_{z, 2}^{2}}$ is indistinguishable from that obtained for $\alpha=0$ for $\beta_{0}<30$. Thus, the equilibrium ensemble average of $\cos ^{2} \theta$, $\left\langle\overline{e_{z}^{2}}\right\rangle$, is usually unaffected by a small induced magnetic moment. For larger values of $\alpha$ we observe that the particle orientation is saturated for smaller values of $\beta_{0}$, and hence the peak position of $\left\langle\overline{e_{z}^{2}}\right\rangle$ moves to lower $\beta_{0}$-values (Fig. 3f).

\subsection{When is the induced magnetic moment relevant?}

To evaluate in more detail the parameters for which the induced magnetic moment is relevant, we compared spectra calculated for $\alpha>0$ for a given value of $\beta_{0}$ with those obtained for $\alpha=0$ and considered the maximum difference between the two curves. In this analysis, we also allowed for an offset and a scaling factor between the two curves deviating from unity. The maximum difference relative to the largest value obtained for $\alpha=0$, hereafter called the relative error, for a single population of particles typically arose from a shift of the position of the peak in the spectrum upon increasing values of $\beta_{0}$. Figs. $5 \mathrm{a}$ and $\mathrm{b}$ show contour plots of the relative error for OM and ACS measurements, respectively.
The quadratic dependence of $\gamma_{0}$ on $\beta_{0}$ causes the induced moment to play a role when $\beta_{0}$ is sufficiently large, even for particles with small values of $\alpha$. Using Fig. 5a and b we observe for particles with $\alpha=10^{-3}$ that the error is smaller than $5 \%$ for $\beta_{0}<50$.

For the multi-core particles with $n_{m}=0.5$ considered in Section 2.4, we found in Eq. (39) that $\alpha=\alpha^{\mathrm{mc}}$ is independent of the size of the particle core. Assuming $\xi=60 \%$, that the magnetization is identical to the saturation of maghemite $\left(M_{\mathrm{s} \text {, maghemite }} \approx 3.7 \times\right.$ $\left.10^{5} \mathrm{~A} / \mathrm{m}\right)$ and that the diameter of the cores fulfill $d_{\mathrm{c}}>17 \mathrm{~nm}$, we obtain $\alpha^{\mathrm{mc}} / \widetilde{K}<0.0153$. The requirement that $\alpha^{\mathrm{mc}}<4 \cdot 10^{-3}$ for the induced moment to be negligible for $\beta_{0}<20$ is then fulfilled for $\widetilde{K}<0.26$. Fig. 5 c shows $\widetilde{K}$ calculated for a prolate ellipsoid as function of the aspect ratio $p_{\mathrm{c}}$ for $\chi=1,3,20$, and $\infty$. It is observed from the figure the requirement $\widetilde{K}<0.26$ is fulfilled when $p_{\mathrm{c}}<4.6$ for $\chi=1$ and $p_{\mathrm{c}}<1.33$ for $\chi=3$, respectively. Thus, for such particles, the induced magnetic moment is only relevant when their aspect ratio deviates significantly from unity.

For single core particles, we found in Eq. (38) that $\alpha \beta_{0}=$ $H_{0} \widetilde{K} / M_{\mathrm{s}}$. The dotted lines in Fig. $5 \mathrm{a}$ and $\mathrm{b}$ show values of constant $\alpha \beta_{0}=10^{-3}, 10^{-2}, 10^{-1}$, and $10^{0}$. As an example of a particle with a low permanent magnetic moment, we take a particle with a permanent magnetisation identical to $5 \%$ of the saturation of maghemite in an applied magnetic field of amplitude $\mu_{0} H_{0}=10 \mathrm{mT}\left(H_{0} \approx 8.0 \times 10^{3} \mathrm{~A} / \mathrm{m}\right)$. Moreover, we take $\tilde{K}=0.16$ corresponding to a prolate ellipsoid with $p_{\mathrm{c}} \approx 2.2$ or $p_{\mathrm{c}} \approx 1.2$ for $\chi=1$ and $\chi=3$, respectively (see Fig. $5 \mathrm{c}$ ). With these values we obtain $\alpha \beta_{0}=H_{0} V_{\mathrm{c}} \tilde{K} / M_{\mathrm{s}} \approx 0.064$. From Fig. 5 we observe that the relative error is below $5 \%$ when $\beta_{0}<50$. At room temperature, the latter requirement corresponds to the particle core diameter being below about $130 \mathrm{~nm}$. For particles with a higher value of $M_{\mathrm{s}}$, we note that $\alpha \beta_{0}$ is inversely proportional to $M_{\mathrm{s}}$ and that $\beta_{0}$ 

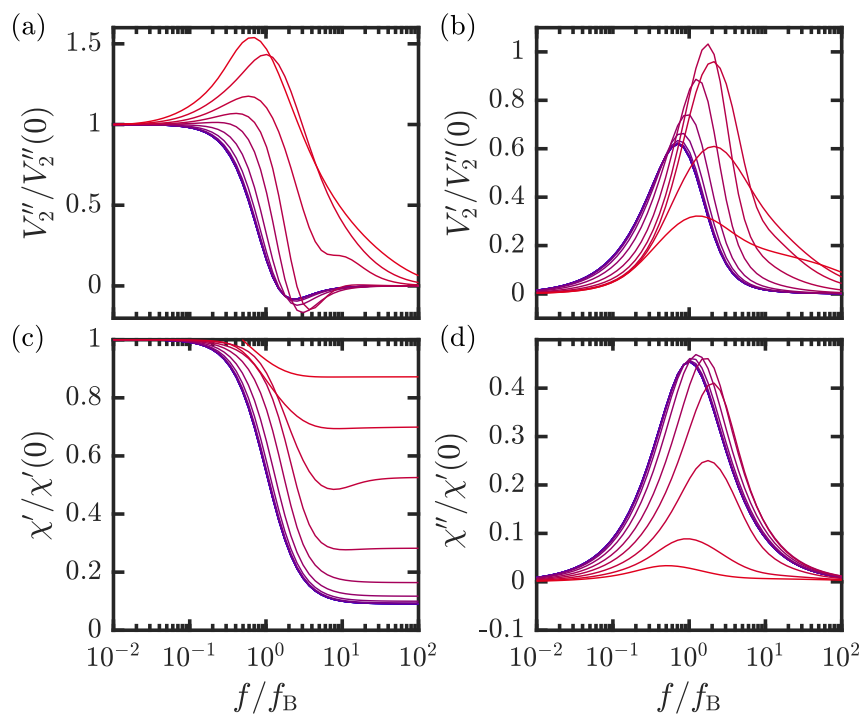

Fig. 4 Optomagnetic (a)-(b) and magnetic susceptibility (c)-(d) from Fokker-Planck calculations (Solid lines) with fixed $\alpha=0.1$ corresponding to $\gamma_{0}=0.1 \beta_{0}^{2}$ with $\beta_{0}$-values logarithmically spaced from 0.01 (blue) to 56 (red) in 16 steps (same values as in Fig. 3). These simulations represent the change of the spectra vs. applied field strength for particles with a fixed geometry. The calculations were performed with $\chi_{\infty}=0$.

is proportional to $M_{\mathrm{s}}$. Therefore, for a constant particle core size, an increase of $M_{\mathrm{s}}$ by a factor of 10 will decrease $\alpha \beta_{0}$ by a factor of 10 and increase $\beta_{0}$ by a factor of 10. As the error contours in Fig. 5a and $\mathrm{b}$ have a slope corresponding to an exponent smaller than -1 , the requirement in the example above would only be fulfilled for particles with sizes slightly below $130 \mathrm{~nm}$. In the experimental study below, we will verify that this assumption is fulfilled.

\section{Experimental studies}

Two particle systems were studied: the multi-core particles NP1 and NP2. Representative TEM images are shown in Fig. 6. Both particle systems consist of cores with diameters $d_{\mathrm{c}}>16 \mathrm{~nm}$ (see Figs. S12b and S13b). The median number of cores per particle for NP1 is 7 according to TEM images (see Fig. S12c). Less than $2 \%$ of the NP1 particles contain one or two cores, in contrast to the NP2 sample were more than $50 \%$ of the particles contain just one or two cores. The particle sizes of the NP1 and NP2 samples estimated from TEM images are similar with sizes ranging from about $50 \mathrm{~nm}$ to $200 \mathrm{~nm}$.

For the particles in this study we assume small aspect ratios $\left(p_{\mathrm{c}}<4 / 3\right)$. Therefore, we can assume $\alpha=0$ in the fitting of the data, i.e., the total magnetic moment $m_{\text {tot }}$ of a particle is assumed identical to the permanent magnetic moment $m$ of a particle.

First, we use previously published methods to independently estimate the distributions of Brownian relaxation frequencies and particle permanent magnetic moments ${ }^{20}$ : the distribution of $f_{\mathrm{B}}$ is estimated from low-field OM and ACS measurements vs. frequency (Section 5.1), and the distribution of $m$ is estimated from low-frequency OM and ACS measurements vs. magnetic field amplitude (Section 5.2). In the analysis, the weighting of the results with the magnetic moment and hydrodynamic particle volume will be taken into account for the two techniques.

Second, we use OM and ACS measurements vs. field and frequency (Section 5.3) to directly estimate the number-weighted bivariate distribution of $f_{\mathrm{B}}$ and $m$ including the correlation between the two parameters. From the resulting distribution, we calculate the appropriately weighted distributions to enable a comparison to the results obtained assuming independent distributions in Sections 5.1 and 5.2.

\subsection{Low-field measurements vs. frequency}

To extract the distribution of Brownian relaxation frequencies or hydrodynamic sizes from low-field measurements, we follow the notation and procedure described in ${ }^{20}$ and analyse the measurements in terms of the weighted lognormal distribution of Brownian relaxation frequencies, $p_{\ln }^{\prime}\left(f_{\mathrm{B}}\right)=\left(f_{\mathrm{B}} / \tilde{f}_{\mathrm{B}}\right)^{-q} p_{\ln }\left(f_{\mathrm{B}}\right)$ where $q$ is an exponent depending on the measurement method and $p_{\ln }\left(f_{\mathrm{B}}\right)$ denotes the number-weighted distribution of Brownian relaxation frequencies. The $\mathrm{OM}$ measurements were analysed using the modified Debye expression with a corrected Brownian relaxation frequency, Eq. (45). The ACS measurements were analysed following the procedure described in $5,30,31$. In ${ }^{20}$ we found the weighting exponents $q^{\mathrm{OM}}=\left(n_{\sigma}+2 n_{m}\right) / n_{\mathrm{h}}$ and $q^{\mathrm{ACS}}=2 n_{m} / n_{\mathrm{h}}$ (see Section 2.4 for definitions). The exponent $n_{\sigma} / n_{\mathrm{h}}$ accounts for the weighting of the optical extinction properties with the hydrodynamic volume in the OM signal and the exponent $2 n_{m} / n_{\mathrm{h}}$ accounts for the weighting of the low-field OM and ACS responses with the magnetic moment squared.

Table 1 shows the parameters $f_{\mathrm{B}}$ and $\sigma_{f_{\mathrm{B}}}$ of a $f_{\mathrm{B}}^{-q}$-weighted lognormal distribution obtained from fits to the low-field OM measurements (Fig. S7 ESI ${ }^{\dagger}$ ) and the low-field ACS measurements (Fig. S8, ESI $\left.{ }^{\dagger}\right)$ with weighting exponents $q^{\mathrm{OM}}=\left(n_{\sigma}+2 n_{m}\right) / n_{\mathrm{h}}$ and $q^{\mathrm{ACS}}=2 n_{m} / n_{\mathrm{h}}$, respectively. Note, that for the case $n_{\sigma}=1$, $n_{m}=0.5$ and $n_{\mathrm{h}}=1$ assumed for the multi-core particles in ${ }^{20}$, we obtain $q^{\mathrm{OM}}=2$ and $q^{\mathrm{ACS}}=1$, i.e., in this case the OM signal is weighted by $V_{\mathrm{h}}^{2}$ and the ACS signal is weighted by $V_{\mathrm{h}}$.

For the NP1 sample, a good fit could be obtained using a monomodal lognormal distribution. However, for the NP2 sample, a bimodal lognormal distribution was needed to obtain a good fit.

The different weighting of the results obtained by the two techniques can cause a significant difference between the two median values. We will later revisit the results when we have estimated the value of $n_{m}$ from analysis of the data vs. both field and frequency in terms of the presented bivariate distribution.

\subsection{Low-frequency OM measurements vs. field}

To extract the distribution of permanent magnetic moments from low-frequency OM measurements vs. magnetic field amplitude we follow a procedure analogous to that for the low-field measurements vs. frequency. The OM measurements were analysed in terms of the weighted lognormal distribution of permanent magnetic moments, $p_{\ln }^{\prime}(m)=(m / \tilde{m})^{r} p_{\ln }(m)$ with $r^{\mathrm{OM}}=n_{\sigma} / n_{m}$ and where $p_{\ln }(m)$ denotes the number-weighted distribution of permanent magnetic moments ${ }^{20}$. The exponent $n_{\sigma} / n_{m}$ accounts for the weighting of the OM signal with the optical extinction proper- 

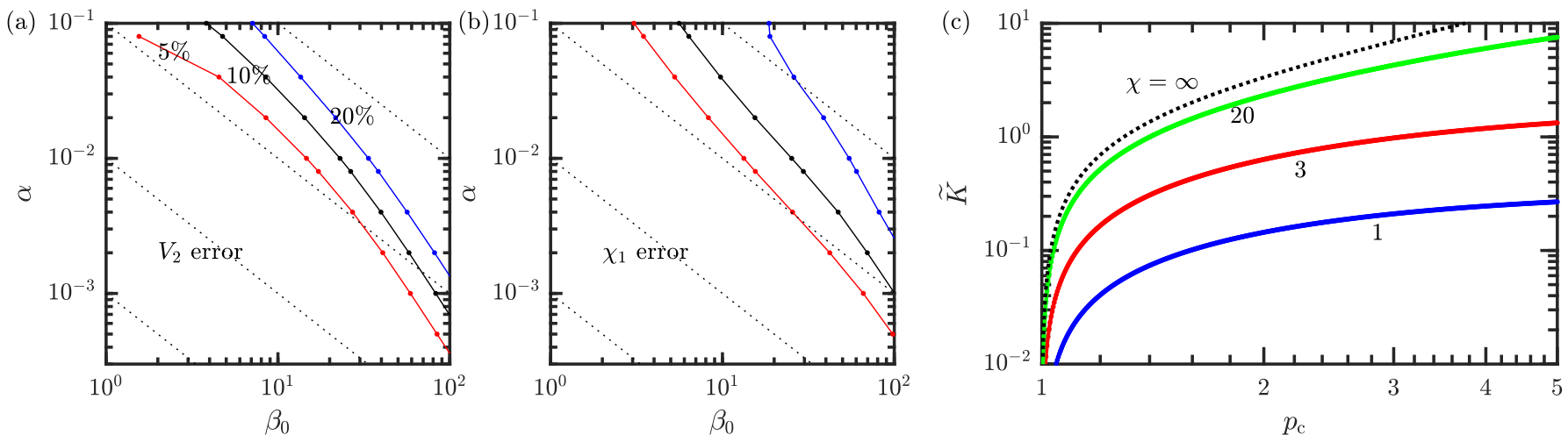

Fig. 5 Contour plots of the maximum difference relative to the maximum amplitude (the error) of scaled and offset spectra for $\alpha=0$ with respect to those calculated for the indicated values of $\alpha>0$ for (a) optomagnetic spectra and (b) magnetic susceptibility spectra. The contours indicate errors of $5 \%$ (red), $10 \%$ (black), and $20 \%$ (blue). The dashed lines shows the contours of constant $\alpha \beta_{0}$ for $\alpha \beta_{0}=10^{-3}, 10^{-2}, 10^{-1}$, and $10^{0}$, respectively. (c) The anisotropy factor, $\widetilde{K}$, vs the aspect ratio, $p_{\mathrm{c}}$, for $\chi=1$ (blue), $\chi=3$ (red), $\chi=20$ (green) and $\chi \rightarrow \infty$ (dotted black).
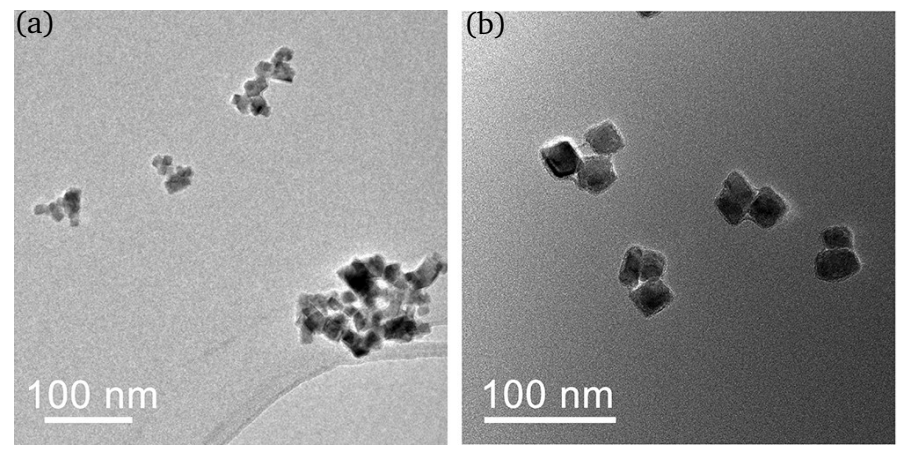

Fig. 6 TEM images of multi-core nanoparticle systems (a) NP1 and (b) NP2.

ties and the magnetic moment. The parameters obtained from fits to the low-frequency OM measurements (Fig. S9, ESI ${ }^{\dagger}$ ) are given in Table 1.

Table 1 Values of $f_{\mathrm{B}}$ and $\sigma_{f_{\mathrm{B}}}$ of $f_{\mathrm{B}}^{-q}$-weighted lognormal distribution fits to low-field OM and ACS data vs. frequency with weighting exponents $q^{\mathrm{OM}}=\left(n_{\sigma}+2 n_{m}\right) / n_{\mathrm{h}}$ and $q^{\mathrm{ACS}}=2 n_{m} / n_{\mathrm{h}}$, respectively. Values of $m$ and $\sigma_{m}$ obtained from $m^{r}$-weighted lognormal distribution fit to low-frequency OM data vs. magnetic field amplitude with weighting exponent $r^{\mathrm{OM}}=n_{\sigma} / n_{m}$.

\begin{tabular}{ccccccc}
\hline & $\begin{array}{c}f_{\mathrm{B}}^{\mathrm{OM}^{\prime}} \\
{[\mathrm{Hz}]}\end{array}$ & $\begin{array}{c}f_{\mathrm{B}}^{\mathrm{ACS}^{\prime}} \\
{[\mathrm{Hz}]}\end{array}$ & $\sigma_{f_{\mathrm{B}}}^{\mathrm{OM}}$ & $\sigma_{f_{\mathrm{B}}}^{\mathrm{ACS}}$ & $\begin{array}{c}m^{\mathrm{OM}^{\prime}} \\
{[\mathrm{aJ} / \mathrm{T}]}\end{array}$ & $\sigma_{m}^{\mathrm{OM}}$ \\
\hline $\mathrm{NP} 1$ & $223(9)$ & $495(2)$ & $0.86(6)$ & $0.931(9)$ & $6.19(5)$ & $0.47(1)$ \\
$\mathrm{NP2}$ & $91(20)$ & $386(105)$ & $0.5(2)$ & $1.63(8)$ & $6.3(1)$ & $0.55(2)$ \\
- & $580(122)$ & $1017(43)$ & $0.6(2)$ & $0.6(1)$ & - & - \\
\hline
\end{tabular}

\subsection{Bivariate magnetic moment and hydrodynamic size dis- tribution fits}

Information on the particle morphology is needed to obtain the weighting of the signal in terms of the particle size. This weighting is required to convert the distributions $p_{\ln }^{\prime}(m)$ and $p_{\ln }^{\prime}\left(f_{\mathrm{B}}\right)$ obtained in section 5.1 and 5.2 to the corresponding number- or volume-weighted distributions. Below, we show that by measuring ACS and OM vs. field and frequency we are able to obtain the number-weighted distribution directly. Further, Table 1 shows that both $m$ and $f_{\mathrm{B}}$ have disperse distributions. The correlation between magnetic moment and Brownian relaxation frequencies can also be extracted by measuring the OM and ACS signals vs. both frequency and field.

$\mathrm{OM}$ and ACS measurements vs. frequency were performed at thirteen different magnetic field strengths $\left(H_{0}\right)$. Figures 7a-d show the data for particle system NP1. The ACS and OM measurements obtained at all field amplitudes were analysed simultaneously using Eq. (37) (ACS) and Eq. (36) (OM) with a twodimensional bivariate number-weighted lognormal distribution with a correlation term as defined in Eq. (40). Free non-linear parameters in the fit were the five parameters of the distribution, $\mu_{m}, \sigma_{m}, \mu_{f_{\mathrm{B}}}, \sigma_{f_{\mathrm{B}}}$, and $\rho$ (see Section 2.4) and the magnetic moment weighting exponent for the OM signal, $r^{\mathrm{OM}}=n_{\sigma} / n_{m}$. Three additional linear fitting parameters were $n, \chi_{\infty}$ and $n z \widetilde{\Delta \sigma} / \widetilde{m}^{n_{\sigma}} / n_{m}$. The ACS measurements were performed at a slightly higher temperature than the OM measurements. To account for this, the temperature dependence of the viscosity of $\mathrm{H}_{2} \mathrm{O}$ was included in the analysis. Further numerical details are given in Section S7, ESI ${ }^{\dagger}$.

The fits of the presented model to the data for the NP1 sample are shown as the lines in Figures 7a-d. Figure 7e shows the obtained 2D distribution, which is described by the fitting parameters given in Table 2. A correlation coefficient of $\rho=-0.82$ was obtained. Inserting the results in Eq. (42), we obtain $n_{f_{\mathrm{B}}}=-0.51$. Assuming that the hydrodynamic volume and the particle core volume are proportional $\left(n_{\mathrm{h}}=1\right)$, we obtain $n_{m}=-n_{f_{\mathrm{B}}}=0.51$. Thus, the fitting results give that the magnetic moment of a particle is proportional to $V_{\mathrm{c}}^{0.51}$. This square root dependence of the magnetic moment on the particle core volume agrees with the expectation for multi-core particles in the limit of many cores ${ }^{4}$. Further, combining $n_{m}=0.51$ with the OM magnetic moment weighting exponent, $r^{\mathrm{OM}}=n_{\sigma} / n_{m}=1.9$, we find $n_{\sigma}=1.0$, which is the value expected when absorption dominates the extinction cross section.

Particle system NP2 was analysed using the same method as described above. The data with fits are shown in Fig. S10 and the obtained fitting parameters are shown in Table 2. However, 
(a)

(b)
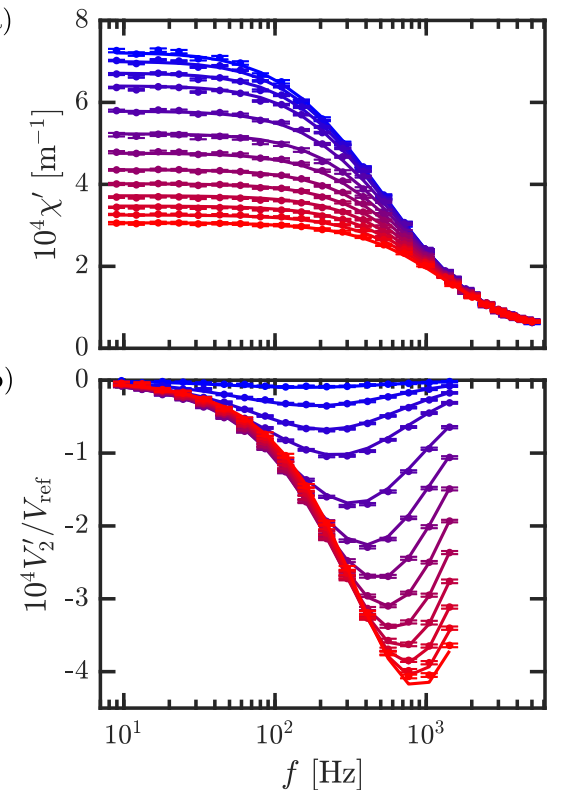

(c)

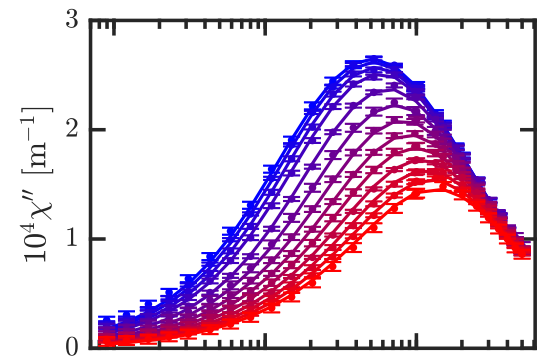

(d)

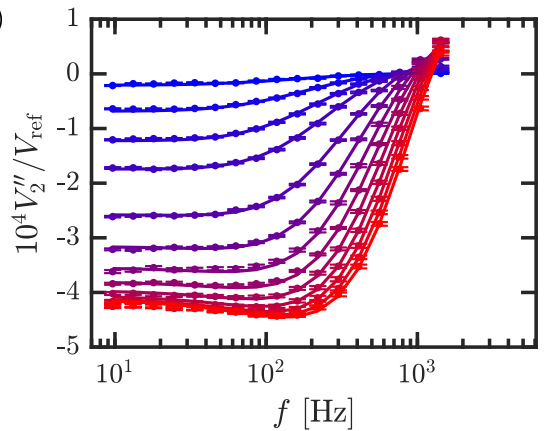

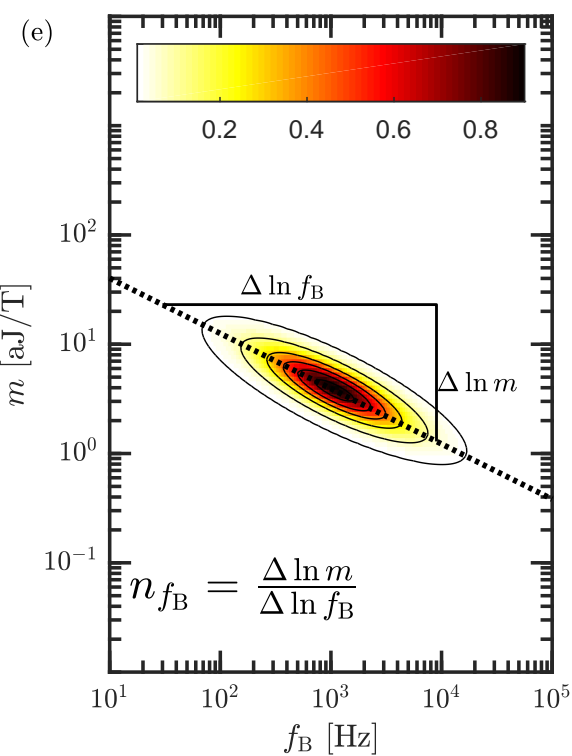

Fig. 7 Measurements vs. field and frequency on the NP1 particles. Panels (a) and (c) show the real and imaginary parts of the ACS data (points). Panels (b) and (d) show the real and imaginary parts of the OM data (points). The solid lines are the fit obtained from simultaneous analysis of all data to a bivariate distribution of Brownian relaxation frequencies and magnetic moments. The colours from blue to red correspond to increasing field amplitudes as given in the Methods section. Panel (e) shows the resulting bivariate distribution function. The distribution is normalised by its maximum value and the contour lines are at $0.01,0.1,0.3,0.5,0.7$ and 0.9 .

Table 2 Parameters of number-weighted bivariate lognormal distribution fits to the OM and ACS measurements vs. frequency and magnetic field. For the NP1 and NP2 samples, monomodal and bimodal distributions were used, respectively. The numbers in parentheses indicate the uncertainty on the last digit obtained from the least squares fitting routine.

\begin{tabular}{cccccccccc|cc}
\hline & $\mu_{f_{\mathrm{B}}}$ & $\sigma_{f_{\mathrm{B}}}$ & $\begin{array}{c}\mu_{m} \\
{[\mathrm{aJ} / \mathrm{T}]}\end{array}$ & & $\sigma_{m}$ & $\rho$ & $\frac{n_{\sigma}}{n_{m}}$ & $\frac{n z \widetilde{\Delta \sigma}}{\widetilde{m}^{n_{\sigma} / n_{m}}}$ & $n$ & $\chi_{\infty}$ \\
& {$[\mathrm{kHz}]$} & & & & & $n_{f_{\mathrm{B}}}$ & {$\left[10^{5}\right]$} & \\
\hline NP1 & $1.074(8)$ & $0.908(3)$ & $3.78(3)$ & $0.513(4)$ & $-0.817(4)$ & $1.88(1)$ & -12.2 & 2.81 & 0.038 & $-0.505(3)$ \\
NP2 & $2.82(8)$ & $1.60(1)$ & $4.00(8)$ & $0.550(8)$ & $-1.00(1)$ & $0.66(3)$ & -155. & 1.82 & 0.039 & $-0.344(4)$ \\
- & $1.337(1)$ & $0.375(1)$ & $4.02(6)$ & $0.360(5)$ & $-0.87(1)$ & $6.1(1)$ & -0.00619 & 1.29 & - & $-0.95(2)$ \\
\hline
\end{tabular}

for this sample it was not possible to obtain a good fit using a mono-modal bivariate log-normal distribution of hydrodynamic sizes and moments, and therefore a bi-modal bivariate log-normal distribution was used.

For both peaks of the bi-variate distributions, $m$ and $f_{\mathrm{B}}$ were found to be correlated with $\rho=-1.0$ and $\rho=-0.87$ and exponents $n_{f_{\mathrm{B}}}=-0.34$ and $n_{f_{\mathrm{B}}}=-0.95$, respectively. Assuming $n_{\mathrm{h}}=1$, we obtain $n_{m}=-n_{f_{\mathrm{B}}}$. Further, considering that $r^{\mathrm{OM}}=n_{\sigma} / n_{m}$, we can estimate $n_{\sigma} \approx r^{\mathrm{OM}} n_{m}$. Inserting the values for the NP2 sample from Table 2, we estimate $n_{\sigma}=0.23$ and 5.8, respectively. These two values differ significantly from each other and from the expectation of $n_{\sigma} \approx 1$ for an extinction cross section dominated by absorption.

\subsection{Discussion}

The fits were performed using the analytical aproximations, Eqs. (46)-(47) and (S33)-(S34), as well as the spectra calculated directly by solving the Fokker-Planck equation. The resulting parameters were found to be identical. This proves that the ana- lytical approximations describe the shapes of the curves sufficient well to be used in fitting routines.

We found that the NP1 particle system behaves according to the multi-core model, and that the core volume is proportional to the hydrodynamic volume. The obtained bivariate distribution provides a correlation between the particle volume and the moment, which is in agreement with the scaling factor obtained for the OM measurements. This is a strong indication that the multicore model provides a valid description of this particle system. This agrees with expectations as the majority of the particle cores are blocked and that the number of cores $N$ is high $(N>2$ see Fig. S12c).

The particles in the NP2 sample, on the other hand, only contain few cores per particle. According to TEM images, 50\% of the particles contain one or two cores (see Fig. S13c). Therefore, caution should be taken when fitting with models assuming the scaling laws described by Eqs. (31)-(33). Moreover, according to the fitting parameters, $20 \%$ of the signal originates from particles with a hydrodynamic diameter above $200 \mathrm{~nm}$. This large span of 
particle sizes can cause the extinction properties to change dramatically over the range of particle sizes causing the scaling of Eq. (33) to be invalid. When the size of a particle is on the order of the wavelength of the light the scattering properties of the particles will change and eventually result in the prolate particles to extinct more light when their long axis is aligned along the magnetic field, thus changing the sign of $\Delta \sigma$ to positive ${ }^{32}$. In fits assuming the scaling in Eq. (33), the exponent $n_{\sigma} / n_{m}$ will therefore be lower to effectively reduce the signal from the largest particles.

For the NP2 sample, we also analysed only the ACS data using a bimodal bivariate distribution (Fig. S11). We obtained $n_{f_{\mathrm{B}}} \simeq-0.4$ for both modes, which is different compared to when fitting to the combined ACS and OM dataset, where one of the modes had $n_{f_{\mathrm{B}}}=-0.34$ and the other had $n_{f_{\mathrm{B}}}=-0.95$. This is a strong indication that the $\Delta \sigma$ scaling as given in Eq. (33) is not valid for this sample. The $n_{f_{\mathrm{B}}}$-values obtained from the ACS fits are slightly lower than expected from the ideal multi-core model. This can be explained by the small number of cores in the particles: When a particle has only two cores, the cores are likely to form chains of aligned magnetic moments. However, an extra core added is likely to position itself adjacent to the dimer such that the total magnetic moment is reduced such that the value of $m$ for $N=3$ is lower than that for $N=2$. This is a more complicated behaviour than the simple $N^{1 / 2}$ dependence for multi-core particles with many cores and indicates that when the the number cores per particle is low, Eq. (32) provides a poor description of the scaling of the magnetic moment with the particle size and fitting the data using this assumption can result in a lower value of $n_{f_{\mathrm{B}}}$.

In the fit we obtained independently the parameters $r^{\mathrm{OM}}=$ $n_{\sigma} / n_{m}$ and $n_{f_{\mathrm{B}}}=-n_{m} / n_{\mathrm{h}}$, which can be related to the particle morphology. The values of these parameters can be used as a control of the validity of the model used. As seen above, the NP1 data could consistently be described with realistic parameters $n_{m} \approx 0.5$ and $n_{\mathrm{h}} \approx 1$. The NP2 data, however, could not be described consistently with realistic values of $n_{m}$ and $n_{\mathrm{h}}$.

For the NP1 sample, the hydrodynamic diameter of the particles can be calculated from the Brownian relaxation frequency using Eq. (6). Using $\eta=0.961 \mathrm{mPa}$ s and $T=298 \mathrm{~K}$, we find the numberweighted median hydrodynamic diameter to $77(1) \mathrm{nm}$ and the corresponding number-weighted mean hydrodynamic diameter to $81(1) \mathrm{nm}$. The number-weighted distribution of hydrodynamic diameters compares perfectly with the histogram of particle sizes obtained from TEM images (Fig. S12a).

Table 2 shows the parameters for the number-weighted distributions. To be able to compare to Table 1 we need to have the same weighting of the distributions. A weighting of a lognormal distribution does not change the width, but only scales the median value. We can estimate the median magnetic moment and median Brownian relaxation frequency with the weighting of the signal we expect for the low-field and low-frequency measure- ments using 20

$$
\begin{aligned}
m^{\mathrm{OM}^{\prime}} & =\mu_{m} \exp \left(\frac{n_{\sigma}}{n_{m}} \sigma_{m}^{2}\right) \\
f_{\mathrm{B}}^{\mathrm{OM}^{\prime}} & =\mu_{f_{\mathrm{B}}} \exp \left(-\frac{n_{\sigma}+2 n_{m}}{n_{\mathrm{h}}} \sigma_{f_{\mathrm{B}}}^{2}\right) \\
f_{\mathrm{B}}^{\mathrm{ACS}} & =\mu_{f_{\mathrm{B}}} \exp \left(-\frac{2 n_{m}}{n_{\mathrm{h}}} \sigma_{f_{\mathrm{B}}}^{2}\right)
\end{aligned}
$$

Inserting the values from Table 2 in Eqs. (54)-(56), we obtain the median values shown in Table 3. Comparing with Table 1, we observe generally a good agreement. For the NP1 sample, the Brownian relaxation frequency is $6 \%$ lower than the value obtained from the low-field fit. The calculations in Eqs. (54)-(56) give the expected values for measurements performed with $\beta_{0} \ll 1$. However, due to the finite measurement fields $\beta_{0} \simeq 1$ for the largest particles and consequently the relaxation response for these particles will be shifted to higher frequencies resulting in a higher median relaxation frequency than would be obtained in the true low-field regime. For the magnetic moment determination, this issue is not relevant, and we found that the median moment obtained from the measurement vs. field and frequency agreed with that from the low-frequency measurements within the uncertainty.

Table 3 The expected median magnetic moment and median Brownian relaxation frequency of low-frequency and low-field fit estimated from the parameters in Table 2

\begin{tabular}{cccc}
\hline & $f_{\mathrm{B}}^{\mathrm{OM}^{\prime}}[\mathrm{Hz}]$ & $f_{\mathrm{B}}^{\mathrm{ACS}}[\mathrm{Hz}]$ & $m_{0}^{\mathrm{OM}^{\prime}}[\mathrm{aJ} / \mathrm{T}]$ \\
\hline NP1 & $213(2)$ & $467(1)$ & $6.21(1)$ \\
NP2 & $272(8)$ & $488(7)$ & $4.88(7)$ \\
- & $451(6)$ & $1022(5)$ & $8.8(1)$ \\
\hline
\end{tabular}

In this work we only used the lowest harmonics in the analysis of the OM and ACS signals. However, the higher harmonics are also directly obtained from the Fokker-Planck calculations and can be used to analyse the data. Martens et al. ${ }^{13}$ used the ratio between higher harmonics in ACS measurements to characterise magnetic nanoparticles similar to the NP1 sample in the present study. They found that they needed to use a distribution of particle sizes to properly describe ACS data. The solution of the Fokker-Planck equation presented here with $\gamma_{0}=0$ is equivalent to the method used by Martens et al. and we obtain the same results (see Section S11, ESI ${ }^{\dagger}$ ). Martens et al., however, did not use a distribution of magnetic moments, which we have found important to properly describe ACS data vs. both field and frequency. Thus, although the model used by Martens et al. does describe their measurements obtained for a single amplitude of the magnetic field, their resulting fitting parameters should be used with care. Their method could be significantly improved if measurements were performed at multiple field strengths.

The ability to obtain directly from ensemble measurements the number-weighted distribution including correlations relies on the non-linear behaviour of the responses. Therefore, the magnetic field strength has to be strong enough to ensure that $\beta_{0} \gtrsim 1$ for 
the particle system under investigation. Consequently, for particles with a small magnetic moment, a large magnetic field amplitude is needed. Also, the frequency range needs to be adjusted to ensure that the Brownian relaxation frequency is within the range of investigated frequencies. Consequently, small particles require measurements at higher frequencies.

\section{Conclusion}

We derived a system of linear equations from the Fokker-Planck equation for particles which can have a permanent as well as an induced magnetic moment. We investigated the influence of the induced magnetic moment on the field- and frequency-dependent magnetic AC susceptibility (ACS) signal and the optomagnetic (OM) signal. For particles with only a permanent magnetic moment $m$ we developed analytical approximations for the fieldand frequency-dependent ACS and OM signals that are valid for $\beta_{0}=m \mu_{0} H_{0} /\left(k_{\mathrm{B}} T\right)<100$.

Using ACS and OM measurements vs. field and frequency we were able to determine the bivariate two-dimensional numberweighted distributions of magnetic moments and Brownian relaxation frequencies. The correlation between the magnetic moment and Brownian relaxation frequency was found to follow a power law with an exponent close to -0.5 . This indicates that the magnetic properties of the particles can be described as a sum of blocked and randomly oriented core magnetic moments with negligible or insignificant exchange interactions. The presented method not only allows for more detailed analysis of the particle systems by examining the change of the spectral shape with increasing field strength, but it also allows for measurements outside the linear region at low magnetic fields. This has the potential to speed up the measurement time significantly, because of the larger signal obtained for measurement at higher field strengths.

\section{Conflicts of interest}

There are no conflicts to declare.

\section{Acknowledgment}

We thank Micromod, DE, for the coating and fractionation in the synthesis of NP2. This work was supported by EU FP7 grant no. 604448-NanoMag.

\section{References}

1 Q. A. Pankhurst, J. Connolly, S. K. Jones and J. Dobson, J. Phys. D. Appl. Phys., 2003, 36, R167-R181.

2 F. Ludwig, D. Eberbeck, N. Löwa, U. Steinhoff, T. Wawrzik, M. Schilling and L. Trahms, Biomed. Tech. Eng., 2013, 58, 535-545.

3 S. Schrittwieser, B. Pelaz, W. Parak, S. Lentijo-Mozo, K. Soulantica, J. Dieckhoff, F. Ludwig, A. Guenther, A. Tschöpe and J. Schotter, Sensors, 2016, 16, 828.

4 F. Ahrentorp, A. Astalan, J. Blomgren, C. Jonasson, E. Wetterskog, P. Svedlindh, A. Lak, F. Ludwig, L. J. van IJzendoorn, F. Westphal, C. Grüttner, N. Gehrke, S. Gustafsson, E. Olsson and C. Johansson, J. Magn. Magn. Mater., 2015, 380, 221226.
5 P. C. Fannin, B. K. P. Scaife and S. W. Charles, J. Magn. Magn. Mater., 1988, 72, 95-108.

6 B. H. Erné, K. Butter, B. W. M. Kuipers and G. J. Vroege, Langmuir, 2003, 19, 8218-8225.

7 K. Enpuku, T. Tanaka, Y. Tamai, F. Dang, N. Enomoto, J. Hojo, H. Kanzaki and N. Usuki, Jpn. J. Appl. Phys., 2008, 47, 78597865.

8 A. P. A., C. Jonasson, K. Petersson, J. Blomgren, D. Ilver, A. Krozer and C. Johansson, J. Magn. Magn. Mater., 2007, 311, 166-170.

9 F. Ludwig, A. Guillaume, M. Schilling, N. Frickel and A. M. Schmidt, J. Appl. Phys., 2010, 108, 033918.

10 F. Ludwig, AIP Conf. Proc., 2010, 1311, 249-254.

11 G. T. Landi, F. R. Arantes, D. R. Cornejo, A. F. Bakuzis, I. Andreu and E. Natividad, J. Magn. Magn. Mater., 2017, 421, 138-151.

12 J. Dieckhoff, D. Eberbeck, M. Schilling and F. Ludwig, J. Appl. Phys., 2016, 119, 043903.

13 M. A. Martens, R. J. Deissler, Y. Wu, L. Bauer, Z. Yao, R. Brown and M. Griswold, Med. Phys., 2013, 40, 022303.

14 T. Yoshida and K. Enpuku, Jpn. J. Appl. Phys., 2009, 48, 127002.

15 H. Remmer, M. Gratz, A. Tschope and F. Ludwig, IEEE Trans. Magn., 2017, 1, 1-1.

16 M. Gratz and A. Tschöpe, J. Phys. D. Appl. Phys., 2017, 50, 15001.

17 T. Klein, A. Laptev, A. Günther, P. Bender, A. Tschöpe and R. Birringer, J. Appl. Phys., 2009, 106, 114301.

18 A. Günther, P. Bender, A. Tschöpe and R. Birringer, J. Phys. Condens. Matter, 2011, 23, 325103.

19 A. Tschöpe, K. Birster, B. Trapp, P. Bender and R. Birringer, J. Appl. Phys., 2014, 116, 184305.

20 J. Fock, C. Jonasson, C. Johansson and M. F. Hansen, Phys. Chem. Chem. Phys., 2017, 19, 8802-8814.

21 M. Donolato, P. Antunes, T. de la Torre, Zardán Gómez de la Torre, E.-T. Hwu, C.-H. Chen, R. Burger, G. Rizzi, F. G. Bosco, M. Strømme, A. Boisen and M. F. Hansen, Biosens. Bioelectron., 2014, 67, 649-655.

22 J. A. Osborn, Phys. Rev., 1945, 67, 351-357.

23 W. T. Coffey, P. J. Cregg and Y. U. P. Kalmykov, Adv. Chem. Phys., 1993, 83, 263-464.

24 NanoMag (EU FP7 grant no. 604448-NanoMag) homepage, http://www.nanomag-project.eu/, Accessed: 2017-09-13.

25 M. Andrés Vergés, R. Costo, A. G. Roca, J. F. Marco, G. F. Goya, C. J. Serna and M. P. Morales, J. Phys. D. Appl. Phys., 2008, 41, 134003.

26 R. Costo, V. Bello, C. Robic, M. Port, J. F. Marco, M. Puerto Morales and S. Veintemillas-Verdaguer, Langmuir, 2012, 28, 178-185.

27 J. Teller, F. Westphal and C. Gruettner, Magnetic nanoparticles having improved magnetic properties, 2010, Patent No. US 7,691,285 B2.

28 A. O. Ivanov, V. S. Zverev and S. S. Kantorovich, Soft Matter, 2016, 12, 3507-3513. 
29 J. Dieckhoff, M. Schilling and F. Ludwig, Appl. Phys. Lett., 2011, 99, 112501.

30 P. Bender, C. Balceris, F. Ludwig, O. Posth, L. K. Bogart, W. Szczerba, A. Castro, L. Nilsson, R. Costo, H. Gavilán, D. González-Alonso, I. de Pedro, L. F. Barquín and C. Johansson, New J. Phys., 2017, 19, 073012.

31 F. Ludwig, C. Balceris, C. Jonasson and C. Johansson, Analysis of ac susceptibility spectra for the characterization of magnetic nanoparticles, IEEE Trans Magn, 2017, (in press, DOI: 10.1109/TMAG.2017.2693420).

32 M. Donolato, P. Antunes, R. S. Bejhed, T. Zardán Gómez de la Torre, F. W. Østerberg, M. Strömberg, M. Nilsson, M. Strømme, P. Svedlindh, M. F. Hansen and P. Vavassori, Anal. Chem., 2015, 87, 1622-1629. 


\section{Graphical abstracts}

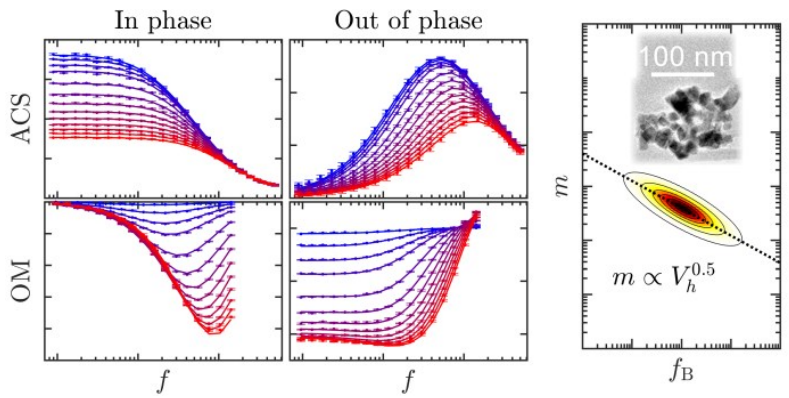

AC susceptibility (ACS) and optomagnetic (OM) measurements vs field and frequency allow determination of the bivariate distribution in moment and size. The obtained correlation provides information on the morphology of the magnetic nanoparticles. 